\title{
Applications of Graphene and Graphene Oxide in Smart Drug/Gene Delivery: Is the World Still Flat?
}

This article was published in the following Dove Press journal:

International Journal of Nanomedicine

\author{
Mojtaba Hoseini-Ghahfarokhi, (D) 1,2 \\ Soroush Mirkiani, (iD ${ }^{3}$ Naeimeh \\ Mozaffari, (iD ${ }^{4}$ Mohamad Amin \\ Abdolahi Sadatlu, ${ }^{5}$ Amir Ghasemi, ${ }^{6,7}$ \\ Somayeh Abbaspour, ${ }^{6}$ Mohsen \\ Akbarian, ${ }^{8}$ Fatemeh Farjadian, ${ }^{8}$ Mahdi \\ Karimi (D) ${ }^{9}$
}

'Nano Drug Delivery Research Center, Kermanshah University of Medical Sciences, Kermanshah, Iran; ${ }^{2}$ Radiology and Nuclear Medicine Department, School of Paramedical Sciences, Kermanshah University of Medical Sciences, Kermanshah, Iran; ${ }^{3}$ Neuroscience and Mental Health Institute, Faculty of Medicine and Dentistry, University of Alberta, Edmonton, Canada;

${ }^{4}$ Research School of Electrical, Energy and Materials Engineering, The Australian National University, Canberra 260I,

Australia; ${ }^{5}$ Department of Engineering and Science, Sharif University of TechnologyInternational Campus, Kish, Iran;

${ }^{6}$ Department of Engineering, Durham University, Durham DHI 3LE, United Kingdom; ${ }^{7}$ Advanced Nanobiotechnology and Nanomedicine Research Group (ANNRG), Iran University of Medical Sciences, Tehran, Iran; ${ }^{8}$ Pharmaceutical Sciences Research Center, School of Pharmacy, Shiraz University of Medical Sciences, Shiraz, Iran; 'Iran Cellular and Molecular Research Center, Iran University of Medical Sciences, Tehran, Iran

Correspondence: Fatemeh Farjadian Pharmaceutical Sciences Research Center, School of Pharmacy, Shiraz University of Medical Sciences, P.o. Box 7|345-I583,

Shiraz, Iran

Tel +987 I 32424I 27 (302)

Fax +987I32424I26

Email farjadian f@sums.ac.ir

Mahdi Karimi

Department of Medical Nanotechnology, Faculty of Advanced Technologies in Medicine, Iran University of Medical Sciences, Tehran, Iran

Email m_karimy2006@yahoo.com

\begin{abstract}
Graphene, a wonder material, has made far-reaching developments in many different fields such as materials science, electronics, condensed physics, quantum physics, energy systems, etc. Since its discovery in 2004, extensive studies have been done for understanding its physical and chemical properties. Owing to its unique characteristics, it has rapidly became a potential candidate for nano-bio researchers to explore its usage in biomedical applications. In the last decade, remarkable efforts have been devoted to investigating the biomedical utilization of graphene and graphene-based materials, especially in smart drug and gene delivery as well as cancer therapy. Inspired by a great number of successful graphene-based materials integrations into the biomedical area, here we summarize the most recent developments made about graphene applications in biomedicine. In this paper, we review the up-to-date advances of graphene-based materials in drug delivery applications, specifically targeted drug/ gene delivery, delivery of antitumor drugs, controlled and stimuli-responsive drug release, photodynamic therapy applications and optical imaging and theranostics, as well as investigating the future trends and succeeding challenges in this topic to provide an outlook for future researches.
\end{abstract}

Keywords: graphene, graphene oxide, drug delivery, gene delivery, cancer therapy, photodynamic therapy

\section{Introduction}

Several shortcomings of traditional therapeutics and low efficacy of drugs for in vivo administration have resulted in the progress of nanobiomedicine and modern drug delivery platforms. These drawbacks include abrupt metabolization and exertion of drugs from the body before operation on their targeted sites, poor solubility of some drugs in aqueous medium, non-specific targeting, low retention effectiveness on deceased regions and adverse effects on normal cells. However, recent progress of nanotechnology and nanomaterials have provided a bright prospect in the biomedicine area, namely a better bio-distribution of drugs, reduced adverse effects on healthy cells, specific selectivity, and higher local therapeutic absorption with the help of newly proposed drug delivery systems. During the last decades, many nanomaterials have been explored by researchers in the nanobiomedicine field including polymeric nanoparticles, micelles, liposomes, mesoporous inorganic nanoparticles, metal oxides, noble materials, carbon nanomaterials etc. ${ }^{1-9}$ Besides the mentioned nanomaterials, the newly proposed two-dimensional nanomaterials have attracted the attention of many scientists because of their unique properties like their very high surface area, biocompatibility, and easy modification.

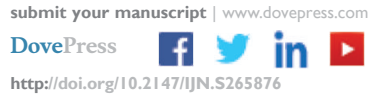


Graphene, the so-called two-dimensional material is a uniformed flat monolayer of carbon atoms, packed tightly into a hexagonal, honeycomb structure. It is the basic structure of other zero-dimensional (fullerene, graphene quantum dots), one dimensional (CNTs) and three dimensional (Graphite) allotropes of carbon. ${ }^{10}$ In this material, each carbon atom is attached to three other carbon atoms by making three hybridized $\mathrm{sp}^{2}$ bonds while one out-of-plane $\mathrm{p}$ orbital provides the electron delocalization network. ${ }^{3}$ The extremely interesting properties of graphene such as its great mechanical strength, high electrical, thermal and magnetic properties, and unique optical properties have made it a potential candidate to be used in several fields of engineering. ${ }^{2-4,11}$ Specifically, the acceptable biocompatibility, very large surface areas, great optical properties together with straightforward biofunctionalization of graphene and its derivatives (graphene oxide (GO) and reduced graphene oxide (rGO)) have engrossed several researchers in several fields of biomedical engineering like drug/ gene delivery and tissue engineering. ${ }^{12,13}$

In 2008, Liu et al $^{14}$ for the first time explored the graphene capabilities in biological systems where they showed the successful non-covalent attachment of a waterinsoluble, aromatic drug (SN38) to the PEGylated graphene oxide sheets by Van der Waals interaction. ${ }^{14}$ Since then, the research on graphene-based materials for biomedical applications, especially drug delivery systems, has grown exponentially demonstrating their high potential in this field. However, being a water-insoluble material limits the biomedical applications of graphene, mostly cell works. Graphene oxide (GO) and reduced graphene oxide (rGO), are functional derivatives of graphene which possess attractive properties like being watersoluble, emitting visible and near-infrared fluorescence as well as having other graphene characteristics important in drug/gene delivery. ${ }^{15}$

Nanoscale graphene-based materials have also been extensively used for designing targeted and stimuliresponsive drug delivery systems through either external stimulus (such as temperature, light, ultrasound waves, magnetic and electric fields), internal stimulus (such as $\mathrm{pH}$, redox and enzymes) or multi-stimuli-responsive drug delivery systems bringing a controlled smart drug delivery with higher bioactivity, better temporal and spatial control at lower dosages of therapeutic agents, together with decreased toxicity and other adverse side effects. ${ }^{16}$ Therefore, functionalized graphene-based materials are promising candidates to be considered for designing smart drug delivery systems and especially for cancer therapy. Furthermore, therapeutic agents like anticancer drugs, proteins, gene and photosensitizers demonstrate a higher efficiency both in diagnosis and treatment of diseases when combined with graphene-based materials. ${ }^{17-19}$

Recently, in a comprehensive study we could review and discuss developments in graphene and graphene oxide; properties, synthesis, and modifications. ${ }^{10}$ In this review paper, we will investigate the most recent progress in biomedical usage of graphene and graphene-based materials, specifically targeted drug/gene delivery, delivery of antitumor drugs, smart and controlled stimuliresponsive drug release. Also, we will explore the developments in photodynamic therapy applications, optical imaging and theranostics based on the graphene materials with a perspective toward the upcoming challenges and trends in this area.

\section{Drug Delivery Applications}

The main goal of designing an optimized drug delivery system (DDS) is to deliver therapeutic agents inside the diseased region in a controllable manner while having fewer side effects on healthy tissues. Due to poor solubility of some chemotherapy drugs in water as well as lack of specificity in drug localization into a tumor, they often have drastic side effects on non-targeted tissues. In the past few decades, the development in nanotechnology has bypassed some difficulties in this area by introducing nano-sized drug carriers with efficient applications in DDS. ${ }^{20}$ Among various nano-carriers, graphene and some derivatives particularly GO have been widely investigated in the field of nanomedicine as bacterial inhibitors, ${ }^{21}$ imaging contrast agents ${ }^{22}$ and drug/gene delivery systems, ${ }^{14,23}$ due to appropriate expanded surface area, biocompatibility and easiness of surface functionalization. ${ }^{24,25}$ Moreover, the high near-infrared (NIR) wavelengths absorbance capacity of graphene nanostructures is providing an opportunity for utilizing them as photo-thermal therapy agents. $^{26,27}$ Therefore, by merging both potentials of carrying drugs and enhanced photo-heat conversion, these graphene-based nanomaterials (GBNs) have a synergistic effect to be applied as heat-responsive nano-sized drug carriers in therapeutic applications like cancer. ${ }^{28-30}$ However, other types of modified graphene-based nanoplatforms loaded with therapeutic agents could be recognized as stimuli-responsive structures for efficient drug 
delivery. ${ }^{31}$ In this regard, a number of studies about stimuli-responsive graphene-based nanoparticles are reviewed in the next section according to types of environmental stimulus (ie, $\mathrm{pH}$, redox, temperature and magnetic field and light). For better judgment, a list of some remarkable studies with their pros and coins are presented in Table 1.

\section{Targeted Drug Delivery}

At present, we are faced with numerous drug delivery systems as well as targeting methods presented through a wide variety of investigations. ${ }^{39}$ Like other nano-systems which have been utilized for drug delivery, graphene-based materials have the potential to be applied for designing targeted DDSs. Zhang et $\mathrm{al}^{40}$ have used a novel strategy using histidine (His) for mediating the hydrothermal expansion of amorphous zinc oxide (a-ZnO) shells on nanoparticles (NPs) of gold (Au-His@a-ZnO). With the aid of carbodiimide crosslinker chemistry, they integrated Au-His@a-ZnO NPs onto the plane of PEGylated GO (PEG-GO). ${ }^{40}$ In this work, Apt@GO@Au-His@a-ZnO@DOX (Apt is aptamer) nanocomposites were created and indicated high carrying capacity of doxorubicin (DOX) along with near-infrared/pHsensitive release of drugs so that the metal-drug complex dissociated to free the anticancer ions of $\mathrm{Zn}^{2+}$ in the low $\mathrm{pH}$ environment (endosome/lysosome of tumor cell). Moreover, they demonstrated fine biostability and aptamerpromoted attaching to lung tumor cells. The specific binding promoted the cellular uptake into EGFR-mutated tumor site (EGFR is epidermal growth factor receptor) in comparison to non-targeted controls. This work employed A549 cells (human pulmonary adenocarcinoma cells) to turmeric mice revealing appreciable targeted drug delivery and high anticancer efficiency of Apt@GO@Au-His@a-ZnO nanocomposites in vivo. The multifunctional nanocomposites (Apt@GO@Au-His@a-ZnO@DOX) exploited in this research led to high efficiency in targeted, photo/chemotherapy to lung tumors. ${ }^{40}$

In another effort, a quantum dot-based GO nanoparticle (GOQD) was prepared in such a manner that the nanoparticle can be a sound nanocarrier potently delivering a drug during cancer chemotherapy. To apply this DDS in cancer therapy, a nanocargo was flourished through conjugating GOQD with the chitosan functionalized folic acid (FA-CH). This group disclosed that the synthesized FA-CH-GOQD is a favorable biocompatible nanocarrier for targeted DOX delivery with high therapeutic efficiency. ${ }^{41}$
A folic acid (FA) targeted system based on Go-coated $\mathrm{Fe}_{3} \mathrm{O}_{4}$ nanocomposites used is for MRI and Dox delivery. The system abbreviated as FA- $\mathrm{Fe}_{3} \mathrm{O}_{4} @$ nGO-DOX could be fruitful and stable material for targeted drug delivery. ${ }^{42}$ The cell viability investigations into $\mathrm{FA}-\mathrm{Fe}_{3} \mathrm{O}_{4} @$ @ $\mathrm{nGO}$ DOX nanoplatforms were conducted and exhibited Cellosaurus cell line/MGC-803 selective uptake. Selective killing of tumor cells was verified as a result of $\mathrm{pH}$-mediated drug release and selective uptake of the nanoparticles through MGC-803 cells using the folic acid receptor.

Also, it has been reported that in support of tumor cell detection plus targeted release of DOX towards HeLa cells, graphene quantum dots and $\mathrm{Fe}_{3} \mathrm{O}_{4}$ nanocomposites conjugated with lectin protein concanavalin $\mathrm{A}$, might be an auspicious candidate. The whole system was abbreviated as GQD-ConA $@ \mathrm{Fe}_{3} \mathrm{O}_{4}$. It has been shown that drug amounts in HeLa cells increase to more than twice when there is an external magnetic field, which is caused by $\mathrm{Fe}_{3} \mathrm{O}_{4}$ incorporation into the nanocarriers. Apart from that, the targeting role of Concanavalin-A in the nanoparticle was certified by proving the vulnerability of HeLa cells to the drug which was $13 \%$ more, compared to normal cells. ${ }^{43}$

An aptamer-conjugated magnetic GO (MGO) nanocarrier was used for targeting delivery of paclitaxel. MGO nanocarriers had formed in consequence of $\mathrm{Fe}_{3} \mathrm{O}_{4}$ attachment on GO layer followed by joining aptamer as a targeting moiety. Furthermore, an anticancer drug, paclitaxel was loaded on the nanocarrier in such a way that this loading as well as in vitro release results unveiled excellent loading possessing high $\mathrm{pH}$-responsive release and entrapment efficiency of $95.75 \%$. By considering cellular toxicity assay, it was found that MGO nanocarriers were biocompatible with the cell death less than $20 \%$ for fibroblast L-929 cells. However, high cell death was reported for paclitaxel and paclitaxel-loaded MGO on MCF-7 tumor cells at different dosages of the drug. Based on the analysis of flow cytometry, it has been discerned that the nanocarrier is capable of binding to MCF-7 cancerous cells. It was deduced that the created superparamagnetic nanocarrier can be a propitious agent for cancer drug delivery. ${ }^{44}$

An improved fluorinated GO (FGO) as an immensely significant drug carrier with the following properties: 1) high capacity of drug loading; 2) switchable fluorescence; 3) water-soluble; 4) controlled and $\mathrm{pH}$-responsive drug release; 5) high NIR absorption; 6) optimized 
photothermal effects; 7) targeted drug delivery; and 8) nanoscale structure was prepared. At the outset, by applying designed oxygen introduction into the nanosheets, FGO was well modified with folic acid as a targeting agent. Additionally, through the adjustment of fluorinated graphene into a nanoscale structure with $\mathrm{SP}^{2}$ carbon, the photothermal performance was bettered. It is noteworthy that the infinitesimal size of FGO is an advantage for endocytosis into cells. DOX was promptly loaded onto FGO-FA (FA is folic acid). FA guarantees target delivery of the nanoparticle to cancer cells with FA receptor and the rest of the particle triggers release of the DOX under intracellular acid condition. ${ }^{45}$

In a three-parted system named GONs-FITC-ATP (GON: graphene oxide nanosheets, FITC: fluorescein isothiocyanate, and ATP: adenosine-5'-triphosphate) energy transfer between GO, FITC and ATP were pointed out. In the light of this article, FITC is substantially quenched by ATP and through drawing a comparison between the quenching of FITC by GONs and FITC-ATP, it was

Table I Advantages and Limitations of Some Remarkable Published Studies About Functionalized Graphene Oxide Nanoparticles

\begin{tabular}{|c|c|c|c|c|}
\hline $\begin{array}{l}\text { Type of } \\
\text { Nanomaterials }\end{array}$ & Advantages & Limitations & $\begin{array}{l}\text { Carriers/ } \\
\text { Drug/ Gene }\end{array}$ & Ref. \\
\hline GO-FA- $\beta C D$ & $\begin{array}{l}\text { - In vitro cytocompatibility } \\
\text { - In vivo tumor growth inhibition }\end{array}$ & $\begin{array}{l}\text { - Needs for immunogenic and pharmacodynamics } \\
\text { studies } \\
\text { - Low stability }\end{array}$ & DOX & 32 \\
\hline NGO-PEG-RB & $\begin{array}{l}\text { - } \mathrm{pH} \text { dependent, suitable for can- } \\
\text { cer therapy } \\
\text { - High specificity }\end{array}$ & $\begin{array}{l}\text { - Complex synthetic methods } \\
\text { - Potential inflammatory effect on lung tissue } \\
\text { - Needs for in vivo study }\end{array}$ & DOX & 22 \\
\hline ADR-GO & $\begin{array}{l}\text { - High drug loading capacity } \\
\text { - } \text { Biocompatible } \\
\text { - } \mathrm{pH} \text { responsive release }\end{array}$ & $\begin{array}{l}\text { - Similarity between free drug and nano-drug in } \\
\text { term of cytotoxicity. } \\
\text { - Needs for in vivo study } \\
\text { - Potential accumulation of the nanoparticle in } \\
\text { spleen }\end{array}$ & $\begin{array}{l}\text { Adriamycin/ } \\
\text { doxorubicin }\end{array}$ & 33 \\
\hline $\mathrm{GN}-\mathrm{CNT}-\mathrm{Fe}_{3} \mathrm{O}_{4}$ & $\begin{array}{l}\text { - } \mathrm{pH} \text {-dependent drug release } \\
\text { - Superparamagnetic } \\
\text { - Efficient uptake by HepG2 cells }\end{array}$ & $\begin{array}{l}\text { - Potential accumulation of } \mathrm{GN}-\mathrm{CNT}-\mathrm{Fe}_{3} \mathrm{O}_{4} \text { in } \\
\text { hepatocyte } \\
\text { - Needs for in vivo study } \\
\text { - Possible immunogenicity }\end{array}$ & $5 F U$ & 34 \\
\hline PEI-GO & $\begin{array}{l}\text { - High transfection efficiency } \\
\text { - Biocompatible } \\
\text { - Sequential gene and drug co- } \\
\text { delivery }\end{array}$ & $\begin{array}{l}\text { - Complex synthetic methods } \\
\text { - Low water solubility } \\
\text { - Needs for animal modeling }\end{array}$ & siRNA, DOX & 35 \\
\hline PEG-GO & $\begin{array}{l}\text { - Without loss of biological activity } \\
\text { - Without enzymatic hydrolysis } \\
\text { - In vitro cytocompatibility }\end{array}$ & $\begin{array}{l}\text { - Needs for protein folding study } \\
\text { - Needs for inflammatory studies } \\
\text { - Needs for in vivo studies }\end{array}$ & $\begin{array}{l}\text { Proteins } \\
\text { ribonuclease A }\end{array}$ & $\begin{array}{l}\text { \& protein } \\
\text { kinase } A\end{array}$ \\
\hline Ti-GO-BMP2 & $\begin{array}{l}\text { - Efficient carrier for therapeutic } \\
\text { protein BMP2 } \\
\text { - High specificity }\end{array}$ & $\begin{array}{l}\text { - Unfolding of the protein in the presence of } \mathrm{Ti} \\
\text { - Needs for immunogenic and pharmacodynamics } \\
\text { studies }\end{array}$ & BMP2 protein & 37 \\
\hline NGO-PEG-SN38 & $\begin{array}{l}\text { - Delivery of water insoluble } \\
\text { anticancer drugs }\end{array}$ & $\begin{array}{l}\text { - Needs for in vivo study } \\
\text { - Possible unwanted generation of protein corona } \\
\text { - Cell disruption in higher concentration }\end{array}$ & $\begin{array}{l}\text { SN38 (CPT } \\
\text { analog) }\end{array}$ & 14 \\
\hline GO-sulfonic acid & $\begin{array}{l}\text { - Co-delivery of multiple drugs } \\
\text { - Remarkably higher toxicity for } \\
\text { cancer cells }\end{array}$ & $\begin{array}{l}\text { - Needs for in vivo study } \\
\text { - Possible immunogenicity }\end{array}$ & DOX \& CPT & 38 \\
\hline
\end{tabular}

Abbreviations: PEG, polyethylene glycol; RB, rituxan (CD20+ antibody); DOX, doxorubicin; ADR, adriamycin/doxorubicin (DOX); FA, folic acid; $\beta C D, \beta$ cyclodextrin; 5FU, 5-fluorouracil; CNT, carbon nanotubes; BMP2, bone morphogenetic protein-2; Ti, titanium; CPT, camptothecin; PEI, polyethylenimine. 
understood that the latter is far weaker due to the formation of supramolecular structures formed by hydrogen bonds and $\pi-\pi$ interactions. In this research, a model of chemical adsorption of ATP molecules on GON surfaces together with partial blocking of FITC quenching by exploring complex characteristic of the GONs-FITC -ATP ternary system was studied. Accordingly, it was stated that in the case of FITC quenching by GONs, ATP has the role of a moderator. Understanding the mechanism of interaction of FITC labeled probe with ATP could be recognized as key in designing targeted systems. ${ }^{46}$

It was elaborated on a targeted drug delivery system based on graphene quantum dot (GQDs) linked to biotin as a targeting module, for the delivery of DOX to tumor cells. It was observed that GQDs were biocompatible and the biotin-linked GQDs loaded with DOX proved to have greater uptake rather than free DOX or GQDs-DOX in A549 cell line. ${ }^{47}$

A system based on decorated magnetic nanoparticles onto GO layered by mesoporous silica was developed for camptothecin (CPT) delivery. The carriers have a capacity of $20 \%$ regarding drug loading and showed a controlled and $\mathrm{pH}$-responsive drug release. In this study, it was realized that the synthesized structures were considerably successful against HeLa cells. Moreover, under suitable magnetic properties along with high adsorption capacity, it was deemed a beneficial and versatile nanoplatform with the aim of tumor chemotherapy using magnetic targeting. ${ }^{47}$

\section{Delivery of Anti-Cancer Drugs}

Among the astonishing properties of graphene and GO nanomaterials, their high surface area makes them an appropriate selection for nanotherapy. Several reports are published on utilizing these materials, for delivery or co-delivery of anti-cancer drugs.

Naturally functionalized GO by chitosan is an appropriate candidate for being used in nanomedicine by good biocompatibility to co-deliver CPT and 3,3'Diindolylmethane (DIM). ${ }^{48}$ As these two drugs possess diverse performances, their co-delivery engenders combined influence upon breast cancer. Moreover, via antiinflammatory assays and hemolysis, the biocompatibility was assayed. The cellular toxicity was determined by 3-(4, 5-dimethyl-2-thiazolyl) -2, 5-diphenyl-2H- tetrazolium bromide (MTT), sulforhodamine B (SRB) and cell death assays toward MCF-7 cell lines together with some other in vivo studies like evaluating the blood biochemical analysis, the biodistribution of the drug, and bioavailability of the drug. Accordingly, it was deduced that loading of the two drugs onto the nanocarrier in conjunction with each other causes the anticancer activity to grow considerably. Besides, in vivo assessment showed that DIM masked the toxic impacts arising from CPT very well.

In another report, Ginsenoside Rh2 (Rh2) which is a new natural-based anticancer agent was treated with amino-acid modified GO (ie, lysine-Go \& arginine-GO). The MTT assay on cancer cells (ie, MDA-MB/breast cancer, OVCAR3/ovarian cancer, and A375/human melanoma) and MSCs/human mesenchymal stem cells were carried out and showed promising anti-cancer activity on cancer cell lines and lower toxicity on MSCs. ${ }^{49}$

Functionalized GO is introduced as a suitable carrier for DDSs. It was displayed that polyvinyl pyrrolidone (PVP) and B-cyclodextrin modified GO (ie, PVP-GO and B-CD-GO) could preserve SN-38, as an anti-cancer drug. The cytotoxic assay on breast cancer/MCF-7 cells showed that the cytotoxicity of free $\mathrm{SN}-38$ was lower than $\mathrm{SN}-38$ loaded on these structures. In comparative studies, PVPGO/SN-38 showed higher masking action rather than $\beta-$ CD-GO/SN-38 and free SN-38.

Embedded graphene quantum dots/GQDs on CMC (carboxymethyl cellulose) hydrogel film showed high capacity in loading DOX and a long release pattern. Further, these films have demonstrated minor toxicity against blood cancer cells (K562) while it was asserted that DOX-loaded $\mathrm{CMC} / \mathrm{GQD}$ nanocomposite could be exploited as a proper anti-cancer material. ${ }^{50}$

In a noteworthy strategy, P-glycoprotein expression and multidrug resistance/MDR problems involved in cancers, specifically breast cancer, were treated by a multiplexed gene silencing system tailored by two molecular beacons (MBs) embedded in GO and loaded with DOX. The nanosystem can release DOX by the low $\mathrm{pH}$ endosomes while up-taken by MDR/breast cancer cells and could silence the MDR1 gene and erythroblastosis virus E26 oncogene homolog 1 gene by MBs hybridizing with target sequences, plus it is capable of hindering DOX efflux transporter of P-glycoprotein expression and causing the progression of MDR breast cancer to desist. ${ }^{51}$

A novel nanosystem of drug delivery comprising GOPRM/SA (PRM is an abbreviation for natural peptide protamine sulfate and SA represents sodium alginate) has been synthesized using layer-by-layer self-assembly technique and electrostatic attraction to make DOX delivery more efficacious. The employed modification resulted in the improvement of GO nanosheets to be more stable and 
dispersible under $\mathrm{pH}$ of the physiological environment. GO-PRM/SA-DOX indicated successful pH-responsive drug delivery behavior. GO-PRM/SA nanocomposites could enter into the cytoplasm of MCF-7 cells because of two features; minute particle size and high water dispersibility. ${ }^{52}$

In controlling mass diffusion drug delivery via hydrogels, GO could be properly utilized. In an experiment, the diffusion of a peptide through a network of GO membranes and the so-called GO-embedded hydrogels were assessed. They posit that size and density of graphene oxide effect on the drug release rate in a way that their optimization could lead to the peptide release rates possessing high efficiency. ${ }^{53}$

CMC-modified graphene acts as a $\mathrm{pH}$-sensitive carrier for DOX delivery. The platform could interact with drug via hydrogen bonding and $\pi-\pi$ stacking. The system showed high antitumor activity while in vitro assessment was performed on cervical cancer/Hela and fibroblast/ NIH-3T3 cells. ${ }^{54}$

Another synthesized drug delivery system (GO-PEG-FA -CPT) has been announced consisting of GO nanomaterials, an anticancer drug (CPT), polyethylene glycol (PEG), and folic acid (FA). This nanosystem disclosed a $\mathrm{pH}$-responsive CPT delivery. By MTT assay on MCF-7 breast cancer cell lines, the antitumor efficacy of the drug delivery system was monitored which was satisfactory for being undertaken. ${ }^{55} \mathrm{In}$ a similar strategy, CPT loaded GO-PEG-FA. The system demonstrated a high drug-loading capacity of $37.8 \%$. In vitro investigations showed a lengthy drug release of 200 hours. It was found that the drug release was more gradual at acidic $\mathrm{pH}$ in comparison to physiological $\mathrm{pH}$. Herein, two cell models of J774 (a high proliferation rate and macrophage phenotype tumor cell) and HepG2 (folate transporter containing human hepatocellular carcinoma cancer cell) were tested. The cytotoxicity of GO-FA varies on different cell types and did not show much cytotoxicity to J774, whereas there was substantial toxicity to HepG2. Moreover, GO-FA/CPT nanocarrier improved the killing of tumor cells by apoptosis. ${ }^{56}$

\section{Controlled and Stimulated Responsive Drug Release $\mathrm{pH}-$ Responsive Graphene-Based DDSs}

It is well-known that there is a somewhat broad range of $\mathrm{pH}$ values between various organs (from about 2 for the stomach to 7 for the mouth) in the body as well as between micro-organisms at subcellular level (lysosomes $\sim 4$ and cytosol 7.4). Particularly, due to the abnormal characteristics of cancer cells and subsequent accumulation of proton in the extracellular matrix of the cells, the regions around tumors have acidic $\mathrm{pH}$ which has motivated the researchers to design pH-sensitive DDSs. ${ }^{57-59}$ The main drug-loading mechanism on GO nanoplatforms is based on simple physisorption either by $\pi-\pi$ stacking or hydrophobic interactions. After exposure to the acidic environment, such drug molecules and the graphene surface links are weakened owing to the protonation of hydrophobic drugs. As a result, drug release had occurred at lower $\mathrm{pH}$ due to hydrophilicity and solubility increment of a drug. ${ }^{60}$

As the pioneering work in pH-responsive DDS with GNBs, Sun et al synthesized theranostic polyethylene glycol grafted GO (PEG-GO) as a nano-carrier of cancer therapy of DOX. They showed the PEG-GO nanostructure releases about $40 \%$ of loaded DOX in an acidic solution $(\mathrm{pH}=5.5)$ after a day while only $15 \%$ of the drug is released in a physiological $\mathrm{pH}=7.4$ over 2 days. Moreover, they conjugated anti-CD20 (Rituxan) antibody to the PEG-GO for a specific bond to B-cells and achieved sharp NIR fluorescence images from the cells. Cytotoxicity assay results showed an increase in cell growth inhibition rate from $20 \%$ to $80 \%$ for non-Rituxan and Rituxan containing PEG-GO/DOX nanostructures. ${ }^{22}$ In another study, poly (2-(diethylamino) ethyl methacrylate) (PDEMAEMA) as the $\mathrm{pH}$-sensitive part was grafted on to GO and loaded by camptothecin (CPT) as an antitumor drug. The $\mathrm{pH}$-triggered delivery of the drug was examined in buffers with $\mathrm{pH} 5.5,7.4$ and 9.0. The PDEMAEMA-GO nanocarriers had the maximum release of CPT in the $\mathrm{pH}$ of 5.5 over two days $(\sim \% 60)$ while in higher $\mathrm{pH}$ solutions CPT release was very negligible. Additionally, the cytotoxicity test revealed that the PDEMAEMA-GO has no toxicity effect on N2a cells in contrast to PDEMAEMA-GO-CPT with $\sim \% 70$ growth inhibitory effect. ${ }^{60}$

To have both targeting therapy and imaging of hepatoma carcinoma cells simultaneously, cyclic arginyl-glycyl-aspartic acid (cRGD) and chitosan as a targeting moiety (RC) were conjugated and so coated on DOX-loaded GO as a drug carrier to form RC-GO-DOX (Figure 1). It is found that the drug release is $\mathrm{pH}$-dependent so that the release process was greater for lower $\mathrm{pH}$ than higher $\mathrm{pH}$. In addition, targeting ability assay that functionalization of fluorescein isothiocyanate (FITC) conjugated RC-GO-DOX endowed it with an 
obvious enhancement of green fluorescence intensity in images of over-expressing integrin $\alpha v \beta 3$ cell line, Bel-7402. ${ }^{61}$

Recently, modified PEG with 2, 3-dimethylmaleic anhydride (DA)-PEG-GO nanocomposite was fabricated to carry and release DOX as a $\mathrm{pH}$-dependent charge reversal DDS. They reported the uptake of PEG-GO-DA in MCF-7 cells elevates under acidic $\mathrm{pH} 6.8$ because of nanocomposite charge switching to positive. Meanwhile, the cell-killing ability of PEG-GO-DA/Dox is improved under this $\mathrm{pH}$. Taking advantage of high NIR absorbance of GO nanostructures, PEG-GO-DA/DOX in combination with $808 \mathrm{~nm}$ NIR laser exhibited more power to destroy the cancer cells. ${ }^{62}$

A novel receptor-mediated nanoplatform of $\mathrm{GO}$ was functionalized by targeting and apoptosing activator agents; tumor necrosis factor-related apoptosis-inducing ligand (TRAIL), provided with DOX to achieve a synergistic antitumor effect. ${ }^{63}$ As shown in Figure 2, after gathering the nanoplatforms near the tumor site, they enveloped the cell surface via binding of TRAIL on the corresponding receptors. Afterward, the Furin cleaves the peptide linker and apoptosis trigger factor; TRAIL induces caspase-mediated apoptosis death. Finally, the DOX contained GOs are internalized into the cytoplasm and under low $\mathrm{pH}$ of endosomes, drug release is commenced. $^{63}$

Using Pluronic F127 (PF127) as a hydrophilic surfactant, PF127/graphene nano-sheets were assembled which can load DOX up to $289 \%$ (W/W) also, release it $56 \%$ and $25 \%$ in acidic and basic conditions, respectively. The result of water-soluble tetrazolium salts (WST) assay showed that even for high concentrations of PF127/graphene nano-sheets, their toxicity is negligible while PF127/graphene loaded DOX nano-sheets with the same concentration brings the MCF-7 cell proliferation inhibitory up to $50 \% .^{27}$

In another $\mathrm{pH}$-responsive system, dual functionalized GO nanocomposite was fabricated as a carrier of DOX. The DDS was equipped by two well-known biostructures: carboxymethyl chitosan (CMC); with high biocompatibility and solubility in water as well as drug release sensitivity to low $\mathrm{pH}$, and lactobionic acid (LA); a disaccharide containing galactose and gluconic acid to target hepatocytes. It was revealed that the drug release profile has $\mathrm{pH}$ dependency so that it reaches up to about $45 \%$ in $\mathrm{pH} 5.8$ against $20 \%$ in phosphate buffer saline (PBS) with $\mathrm{pH} 7.4$ after 72 hours. Moreover, by coupling FITC as a tracker of the nanocarrier, it was found that this DOX DDS platform can target hepatocellular carcinoma SMMC-7721 cells more than L929 cells. $^{64}$

RGD-linked GQDs containing DOX had proven to be a potent tool to carry the drug and monitor the drug release pattern into cells simultaneously. The RGD-GQD-DOX nano-carriers indicated acceptable biocompatibility, high drug release capability in acidic $\mathrm{pH}(60 \%$ vs $13 \%$ in 5 and $7.4 \mathrm{pH}$, respectively), cytotoxicity effects on DU-145 and PC-3 prostate tumor cells as well as photoluminescence ability to track drug release into the cells. The fluorescence images exhibited that DOX accumulation had occurred in the cell nucleus after 16 hours of incubation. $^{65}$

Application of the GQDs with fluorescent emission capability along with chitosan xerogel coating by loading sodium salicylate (SS) as a drug for wound healing and inflammation treatment has been well studied before. ${ }^{48}$ They revealed that the nano-xerogel has interesting fluorescent properties so that in vitro and in vivo bio-imagings by three different emission colors of blue, green and red which are excited by various wavelengths were well monitored by UV-Vis spectroscopy. In contrast to the previously introduced system related to DOX delivery, this system released more $\mathrm{SS}$ in higher $\mathrm{pH}$ owing to the negative charge of the drug. ${ }^{64}$

By combining magnetic NPs, PEG, chitosan and GO nano-sheets (mGOC-PEG), a DDS with controllable and pH-responsive delivery of both irinotecan (CPT-11) and DOX have been achieved. Based on the position of the magnet under the U87 human glioblastoma cells, mGOCPEG/DOX showed meaningful lethal effect. ${ }^{66}$

Exploiting the anticancer effect and $\mathrm{pH}$ responsiveness of poly(vinylpyrrolidone) nanoparticles, the exfoliated $\mathrm{GO}$ nanosheets were prepared. The GO-PVP-NPs affected the mitochondrial enzymatic activities, proliferation of the cells which may dominantly be attributed to the generation of ROSs. Additionally, the higher release of DOX from the exfoliated GO nanosheet in a low $\mathrm{pH}$ and hypoxic environment demonstrated a great potential to treat the tumors. ${ }^{67}$ In another study, it is shown that modifications of $\mathrm{GO}$ by peptide protamine sulfate (PRM) and sodium alginate (SA) lead to better water dispersibility and stability and also lower protein absorption. A pH-responsive release pattern of DOX and higher cytotoxicity on $\mathrm{MCF}-7$ cells than GO-DOX are the remarkable properties of the novel nanocarriers. ${ }^{52}$

The nanocomposite of carboxymethyl cellulose (CMC) hydrogel and graphene quantum dots (GQD) with the improved ability for swelling in vitro and $\mathrm{pH}$-sensitive 
A

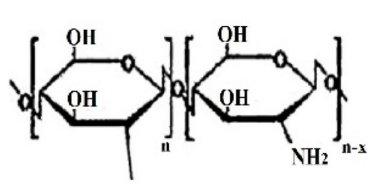

Chitosan

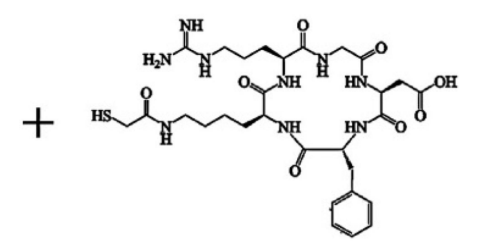

cRGD peptide
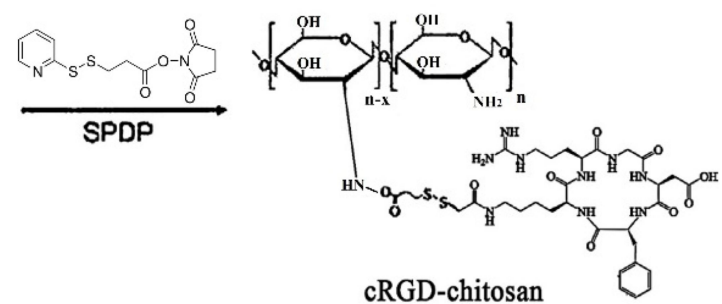

B

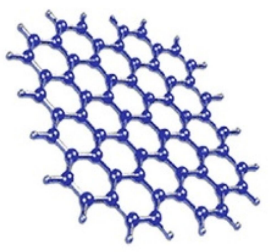

Graphene Oxide (GO)

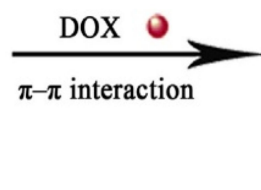

Graphene Oxide-Doxorubicin (GO-DOX)

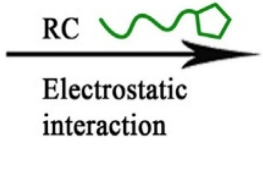

Electrostatic interaction

Figure I Schematic of (A) cRGD conjugated to chitosan (RC) and (B) RC-GO-Dox nanocarrier.

Note: Copyright @2014. Elsevier. Reproduced from Wang C, Chen B, Zou M, Cheng G. Cyclic RGD-modified chitosan/graphene oxide polymers for drug delivery and cellular imaging. Colloids and Surfaces B: Biointerfaces. 2014; 122: 332-340. ${ }^{61}$

release of DOX have been introduced as a theranostic nanoagent for the K562 cancer cells. ${ }^{50}$ In a similar work, the GQD-cross-linked chitosan was loaded by a model drug (sodium salicylate) and encapsulated in CMC. The nanocomposite in the gastrointestinal tract conditions showed high stability and controlled release pattern of the drug. ${ }^{68}$ Focusing on the fluorescent properties of GQDs, the DOX-GQD-RGD was developed to real-time screening of the cellular uptake process as well as drug release location vs the time. The nano-DDS caused the delivery of the DOX into the nucleus of the U251 glioma cells which was followed by increases in cell death. ${ }^{69}$

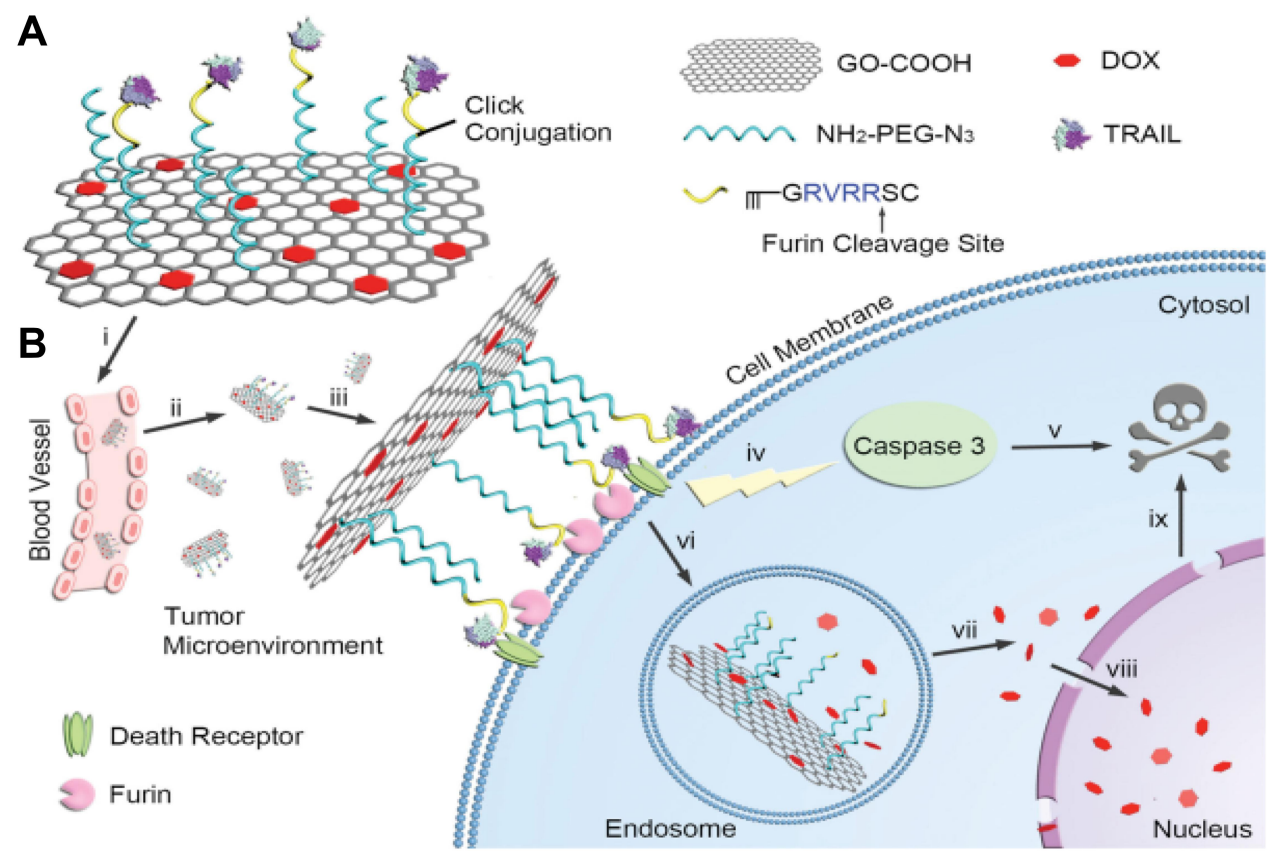

Figure 2 Scheme of TRAIL/DOX-fGO nanoplatform. (A) Main components of TRAIL/DOX-fGO, and (B) Step by step activity of TRAIL/DOX-fGO, from vessel administration to drug release in the cell nucleus.

Note: Copyright @2015. John Wiley and Sons. Reproduced from Jiang T, Sun W, Zhu Q, et al. Furin-Mediated Sequential Delivery of Anticancer Cytokine and SmallMolecule Drug Shuttled by Graphene. Advanced Materials. 2015; 27 (6):1021-1028. ${ }^{63}$ 
In the field of bone cancer treatment, graphene oxide (GO)/hydroxyapatite (HAP)/chitosan (CS) composite was loaded with cisplatin (CDDP). This structure was applied to hinder osteosarcoma cells development and enhance osteoblast cells growth. The GO/HAP/CS-3/CDDP nanocomposites revealed higher cytotoxicity against cancer cells while they showed high viability on osteoblast-like cells and a promising application as a replacement for cancer-affected bone tissues. ${ }^{70}$ In breast cancer therapy, camptothecin (CPT) chemo drug and magnetic nanoparticles were attached to reduced graphene oxide surface, which was cross-linked with 4-hydroxycoumarin (4-HC) photosensitizer through an allylamine (AA) linker. The CPT-loaded MrGO-AA-g-4-HC exhibited superior toxicity effect towards the breast cancer cells in comparison with normal cell lines, resulting in remarkable cancer cell death. ${ }^{71}$ Another study used modified graphene oxidemethyl acrylate (GO-g-MA) conjugated with folic acid in which paclitaxel (PTX) was attached to GO-g-MA/FA nanocarrier by hydrophobic interaction and $\pi-\pi$ stacking. GO-g-MA/FA-PTX nanocarrier revealed 39\% cytotoxic effect that inhibited breast cancer cell growth and decreased size of the tumor. In addition, this system effectively alleviated the mitochondrial dysfunction during breast $^{\text {cancer. }^{72}}$ In the cervical cancer field, $\kappa$ Carrageenan-loaded graphene oxide in conjunction with biotin was applied for cervical cancer treatment. The anticancer drug doxorubicin was grafted on GO- $\kappa$-car-biotin and demonstrated substantial cell death of cancer cells. ${ }^{73}$ In order to improve the curative and permeability of bioactive materials in a drug delivery system, proteinpolymeric carriers have been used. In a recent study graphene oxide was functionalized by means of egg white protein of ovalbumin (OVA) and polymethyl methacrylate (PMMA) and was loaded with doxorubicin on this DDS. The OVA-PMMA-GO-DOX system revealed successful loading and controllable release of the drug. ${ }^{74}$

In a recent study, ultra-small SPIONs were grown on the GO nanosheets and finally were loaded by a modified form of DOX (CAD). The nano-sized theranostic agent proved a high antitumor effect and high-resolution MR imaging in-vivo. ${ }^{75}$

More recently, PEG4000 as a hydrophilic molecule was grafted on the GO nanosheets to elevate the biocompatibility of GO. The DOX release efficiency of the nanosheets was higher for lower $\mathrm{pH}$ than neutral $\mathrm{pH}$ due to H-bonding changes between the drug and nanocarrier. ${ }^{76}$ Beside DOX and CPT, indomethacin ${ }^{77}$ and 5-fluorouracil ${ }^{34}$ are also loaded in GBNs.

\section{Redox-Triggered Drug Release of Graphene}

Exploiting of hydrophilic structures such as PEG and poly (vinyl alcohol) (PVA) to attain further biocompatibility and prolong circulation time in blood have been extensively explored by many investigators. ${ }^{78}$ Nevertheless, the application of these polymers as coating structures can disturb the drug delivery rate due to surface diffusion barriers $^{79}$ and so cause a decline in the release efficiency. On the other hand, the concentration of glutathione (GSH) as the main intracellular redox regulator, is about $2 \mu \mathrm{M}$ and $10 \mathrm{mM}$ for extra- and intracellular environments, respectively. Interestingly, reduced glutathione (GSH) level inside some types of cancer cells rises at least fourfold compared to normal cells. ${ }^{80}$ Therefore, it seems that this significant difference in GSH level can be an appropriate factor to design DDSs. A combination of DDS nanoplatform by coating with biocompatible polymers and redox-sensitive linkage provided a system with ideal criteria of such systems. Redox responsive NGBs have also interested in designing DDSs. In an effort, PEG was linked to GO nanocarrier via disulfide (SS) linkage (NGOSS-mPEG) while antitumor drug DOX was attached (Figure 3). Disulfide linkage is susceptible to cleavage due to the high intracellular level of GSH which induces drugs to release at a higher rate in the cell. In the absence of GSH, less than $35 \%$ (over 48 hours) and only $5 \%$ (over 72 hours) of DOX left the carrier surface at $\mathrm{pH} 5.5$ and 7.4, respectively, while the drug release percentage increased up to 55 and $10 \%$ upon adding $10 \mathrm{mM} \mathrm{GSH}$. Furthermore, enhanced drug release due to S-S bond cleavage induced more growth inhibitory effect on $\mathrm{HeLa}$ cells. ${ }^{81}$

Another PEI-modified GO was applied in redox stimulated delivery by introducing disulfide linkage to PEI and conjugating DOX to this moiety. Furthermore, silver nanoparticles were loaded by GO surface. The intracellular GSH further stimulated drug release by cleavage disulfide bond. Moreover, the presence of silver nanoparticles made monitoring of this material uptake in HeLa cells possible via surface-enhanced raman scattering (SERS) technique. ${ }^{82}$

A novel PEGylated GO, grafted by poly(methacrylic acid) (PMAA) and crosslinked with cystamine as disulfide linkage was introduced to carry DOX. They found PMMA crosslinking on PEG-GO can significantly prevent premature drug release until the SS bond is cleaved by GSH. In pH 5.0 
and $10 \mathrm{mM} \mathrm{GSH}$ condition, the releasing rate was six times faster than $\mathrm{pH} 7.0 .^{83}$ Emphasizing on biocompatibility and controllable drug release of nano-carriers, GO was reduced by 2-chloro-3', $4^{\prime}$-dihydroxyacetophenone quaternized poly (ethylene glycol)-g-poly(dimethylaminoethyl methacrylate) (rGO/QC-PEG). Then, rGO/QC-PEG was loaded with DOX and the complex was fenced by disulfide cross-linking of thiol grafted pluronic (Plu-SH). A schematic of the release mechanism of the prepared nanocarrier is presented in Figure 4.

As shown in Figure 4, under acidic conditions DOX is detached from $\mathrm{rGO} / \mathrm{QC}-\mathrm{PEG}$ uncontrollably. By adding redox-sensitive Plu-SH to $\mathrm{rGO} / \mathrm{QC}-\mathrm{PEG}$, the drug is embayed by Plu-SH and is not released even in the acidic condition. Once the complex is faced with high GSH conditions, disulfide bonds of Plu-SH are broken and so entrapped drugs are released into the environment. This can be resonated in the presence of both acidic and high GSH level simultaneously. ${ }^{84}$ In order to exploit the GO nano-sheets as a powerful theranostic agent activated by redox stimuli, a photosensitizer; chlorine6 (Ce6) was conjugated to GO nano-sheets via a disulfide linker to obtain GO-SS-Ce6. They declared that GO-SS-Ce6 has notable Ce6-related fluorescence intensity while disulfide bonds were cleaved by reductive agent dithiothreitol (DTT). The flow cytometry and confocal laser scanning microscopy results proved a high intracellular penetration of GO-SSCe6 nanostructures into A549 cell line compared with free Ce6. The GO-SS-Ce6 was able to kill about $80 \%$ of A549 cells exposure with $670 \mathrm{~nm}$ diode laser irradiation while free Ce6 has smaller lethal effect in the same condition. ${ }^{85}$

Active targeting of GO nanosheets by hyaluronic acid (HA) via a redox-sensitive linkage imparts simultaneously some extraordinary properties to the nanosheets such as selective accumulation, higher stability in the bioenvironment, drug-loading capacity, NIR absorption and better response to the glutathione presence. ${ }^{86}$

By conjugating $\mathrm{Ce} 6$ as a photodynamic therapy agent to amine group of GO-(NSSNH2)-PEG, a nanocomposite was achieved which could transport Ce6 into the cells more than free Ce6. Moreover, it showed a redoxsensitive release pattern in the presence of glutathione. The animals treated by the nanocomposite had a great accumulation of $\mathrm{Ce} 6$ in the tumor while the liver of other groups exhibited the highest uptake of it. ${ }^{87}$

In a more recent study, gefitinib (Gef) as a drug for lung cancer was linked to $\mathrm{HA}$ functionalized $\mathrm{GO}$ nanosheets. The NGO-SS-HA-Gef nanocarrier showed significant responsiveness to the high level of GSH in A549 lung cancer cells due to the disulfide linkage. ${ }^{88}$ In another study, a redox responsive prodrug (PEG-PCL-SSDOX) was coupled to the GO nanosheets to elevate biostability and accelerate the drug release into the cancer cells. In vivo results exhibited lower systematic toxicity and higher antitumor effect for groups treated by the nanohybrids. ${ }^{89}$

\section{Thermo-Sensitive Drug Delivery by Graphene}

Another strategy to design an efficient DDS can be based on response to temperature changes, particularly for polymeric nanoplatforms. ${ }^{90}$ In this regard, poly (N-isopropylacrylamide) (PNIPAM) has been known as a suitable thermo-sensitive polymer whose lower critical solution temperature (LCST) is about $32^{\circ} \mathrm{C}$ in water. ${ }^{91}$ For instance, GO-PNIPAM was reported as ibuprofen (IBU) contained nanocomposite responsive to changes of the environment temperature. ${ }^{92}$ They reached a large drug loading percentage of $280 \mathrm{wt} \%$ which could be caused by the interaction between a carboxylic part of IBU and $-\mathrm{NH}$ of PNIPAM. Additionally, a long polymeric chain of PNIPAM could keep the drug in its porous structure.

Introducing polymeric NPs including PNIPAM-g-PEO on GO was performed to have a thermo-sensitive nanoplatform working as a protein inhibitor and platelet for adhesion of therapeutic agents in which DOX is tested within. Other than thermoresponsivenesss this nano-hybrid showed high lethal effect on HepG2 cells. ${ }^{93}$

In another study drug loading and release profile of CPT were assessed on PNIPAM-g-GO which exhibited a temperature-dependent manner because a phase transition occurred from hydrophilic to lipophilic at $33^{\circ} \mathrm{C}$. The PNIPAM homopolymer synthesized by the ATRP method was grafted to GO via click chemistry. A noticeable anticancer effect was observed while CPT-loaded PNIPAMg-GO were assessed in vitro with A5RT3 cancer cell line. ${ }^{94}$

A salep modified GO (SMGO) comprising of branched PNIPAM copolymerized with acrylic acid (AA) P (NIPAM-co-AA) as a thermo/pH responding to DOX nanocarriers was introduced. Release tests of DOXloaded nanostructures also showed that maximum drug release happens in acidic $\mathrm{pH}$ of 5.0 and temperature at $42^{\circ} \mathrm{C}$ which confirmed the thermo/pH sensitivity of the nanostructures. In vitro cytotoxicity test of HeLa cells uncovered that SMGO modified P(NIPAM-co-AA) is 


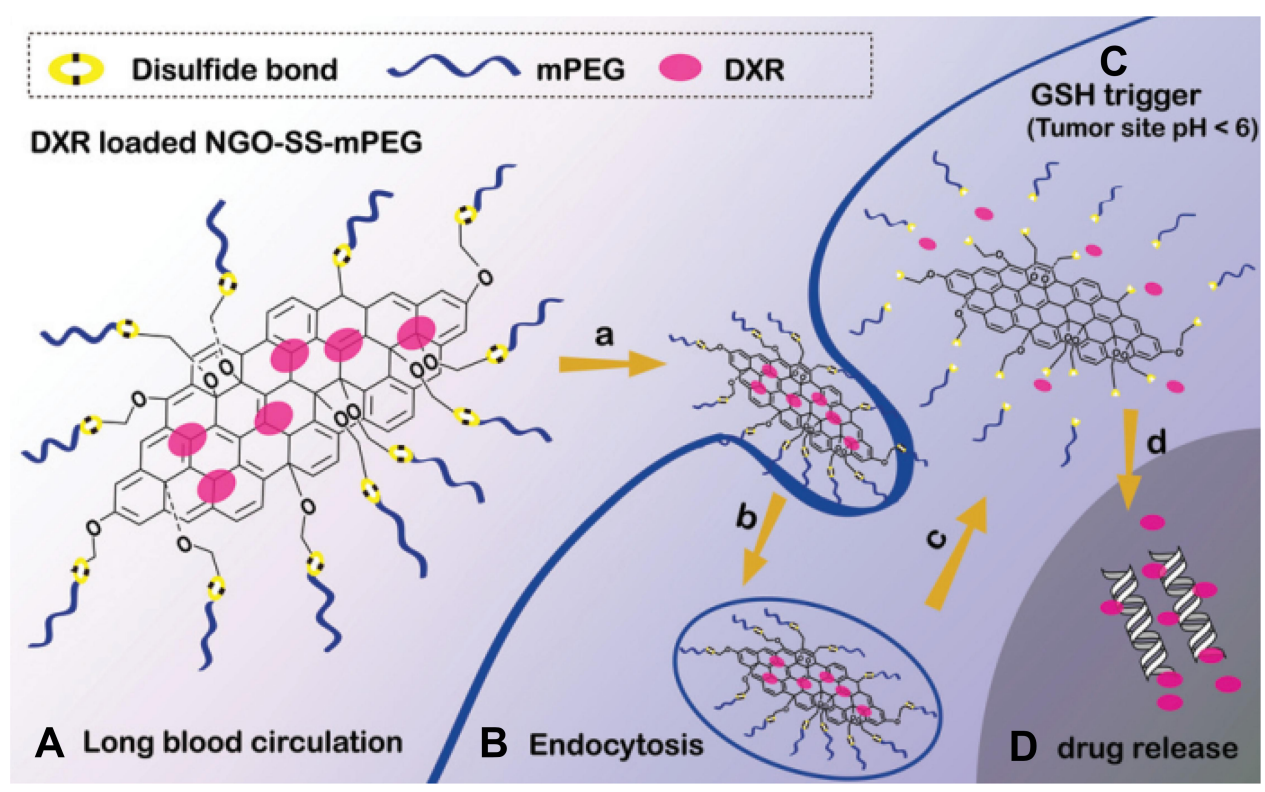

Figure 3 (A) NGO-SS-mPEG in extracellular environment (low GSH level), (B) it is internalized into the tumor cell (high GSH level), (C) linkage is cleaved, and (D) DOX is released.

Note: Copyright @2012. John Wiley and Sons. Reproduced from Wen H, Dong C, Dong H, et al. Engineered redox-responsive PEG detachment mechanism in PEGylated nano-graphene oxide for intracellular drug delivery. Small. 2012; 8 (5): 760-769. ${ }^{81}$

biocompatible as well and can have a significant role in cancer cell killing followed by DOX loading. ${ }^{95}$

Chemically reduced GO nanosheets were functionalized by chitosan followed by copolymerization with NIPAM and PEG-diacrylate as a crosslinker. The synthesized nanogel was loaded by DOX and released the drug in a thermo-responding manner by near infrared (NIR) laser $808 \mathrm{~nm}$. Heat production due to NIR absorption caused the water to squeeze out of nanogels which resulted in shrinkage and size reduction and

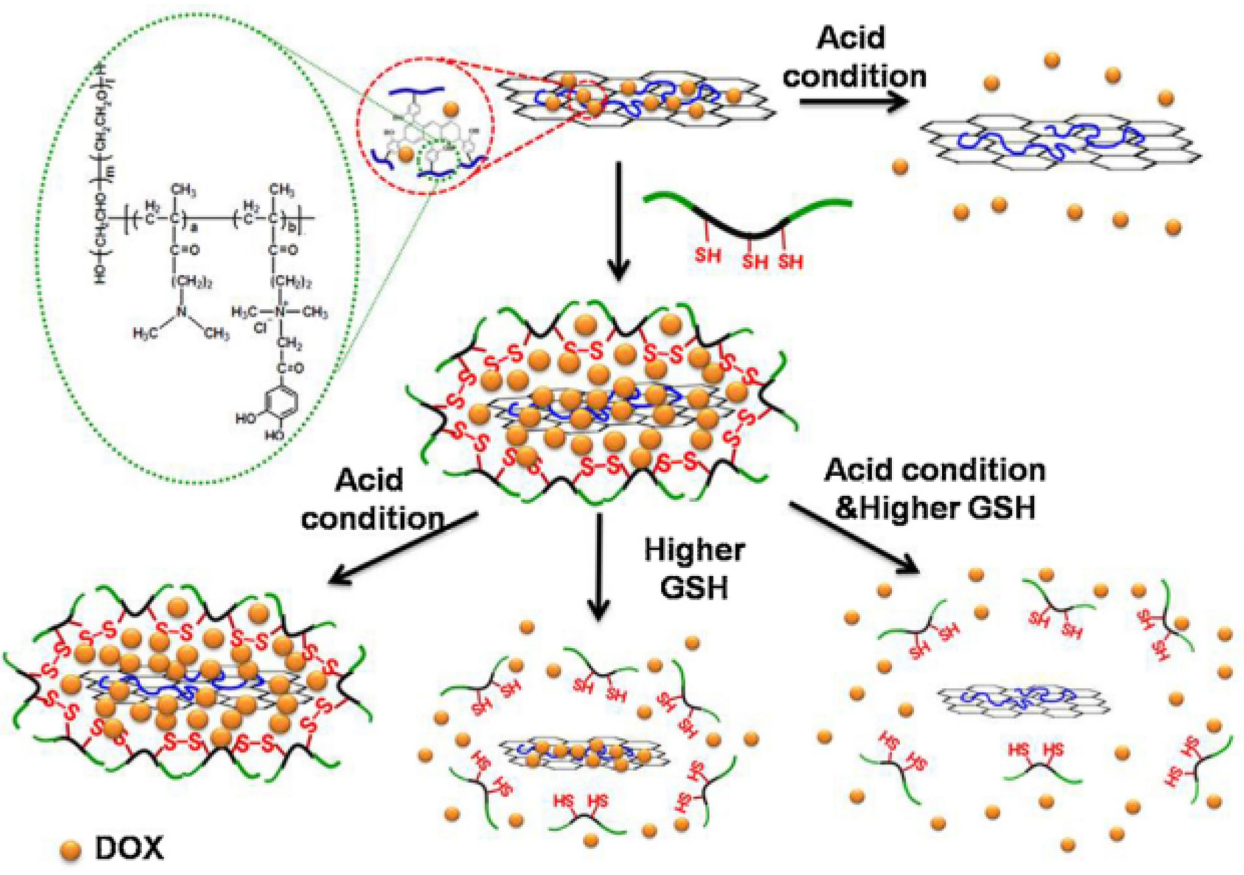

Figure 4 The mechanism of DOX release process in acidic and high GSH level conditions from rGO/QC-PEG and rGO/QC-PEG/Plu-SH .

Note: Copyright C2013. Elsevier. Reproduced from AI-Nahain A, Lee SY, In I, et al. Triggered pH/redox responsive release of doxorubicin from prepared highly stable graphene with thiol grafted pluronic. Int J Pharm. 20I3; 450 (I): 208-217. ${ }^{84}$ 
stimulate the thermo-responsive nanogels to release DOX in the temperature range at $37-42^{\circ} \mathrm{C} .{ }^{96}$

In a more recent study, the quadruple-responsive nanocarrier was developed which could be stimulated by multiple factors like $\mathrm{pH}$, temperature, redox, and NIR irradiation. The smart nanocarrier was loaded by rhodamine $\mathrm{B}$ and for various circumstances it was found that remarkable release occurred after applying multiple stimuli. ${ }^{97}$ As a dual drug nanocarrier, two-faced GO nanosheets grafted by PNIPAM/PCL were prepared. The novel nanoparticles had the potential to load simultaneously hydrophobic (quercetin) and hydrophilic (5-FU) drugs and showed a thermos-responsive release profile. ${ }^{98}$

Recently, it is found that Poly(N,N-diethyl acrylamide)/functionalized GQD hydrogels have a smart behavior against the temperature changes so that the release of the DOX remarkably increases near the physiological temperature. In-vitro and In-vivo results revealed that the smart nanohydrogel has great potential in the treatment of invasive tumor cells like melanoma. ${ }^{99}$

\section{Magnetically Released Drug from Graphene Nanocarriers}

Magnetic nanoparticles have received great attention for drug delivery purposes. ${ }^{100}$ Owing to suitable permeability of electromagnetic fields into the body, they can be used as external stimuli to release drug in DDSs. In order to accumulate and deliver a drug locally, a magnet is located near the desired site of treatment such as tumors by special tools like a conical piece of iron or typical pole shoes. Indeed, the magnetic NPs can indirectly enhance the therapeutic efficiency of GO-based DDSs by further accumulation of drug-loaded NPs in the targeted area ${ }^{101,102}$ or synergistically improvement of cell killing due to magnetic hyperthermia effect. ${ }^{103}$

It has been shown that iron oxide NPs-decorated PEG-GOs (IONP-GO-PEG) congregate where they are affected by an external magnetic field. From the other point of view, DOX loaded in this NPs can be released in greater quantities near tumor $\mathrm{pH}(\sim 5.0)$. In addition to utilizing magnetic properties of IONPs for magnetic resonance imaging (MRI), they caused the death of more 4T1 cells located close to the magnet where IONP-GO-PEGs/DOX was more accumulated compared to regions far from the magnet. ${ }^{104}$

A novel anticancer drug carrier has been presented; polyvinylpyrrolidone (PVP) and PVA stabilized iron oxide graphene whose release manner was sensitive to low $\mathrm{pH}$ and AC magnetic field (ACMF). Although both PVP and PVA stabilized iron oxide graphene containing
DOX and paclitaxel had a lower killing effect on HeLa cells than free DOX or paclitaxel, exposure of the cells to ACMF and PVP and PVA stabilized iron oxide graphene DOX or paclitaxel simultaneously could increase the cell death up to 90 and $93 \%$, respectively. ${ }^{103}$

To combine gene therapy with anticancer drug delivery, chitosan functionalized magnetic graphene (CMG) NPs were synthesized ${ }^{105}$ and reported that these biocompatible NPs were capable of releasing DOX under $\mathrm{pH} 5.1$ at a higher rate than 7.4. Besides high potential use as an MRI contrast agent, a green fluorescent protein (GFP) encoder plasmid was effectively transfected into cells in in-vitro and in-vivo in addition to DOX release. ${ }^{106}$

It has been demonstrated that $\beta$-cyclodextrin modified magnetic $\left(\mathrm{Fe}_{3} \mathrm{O}_{4}\right)$ graphene oxide (MGC) not only could be used in local drug delivery owing to superparamagnetic properties but is able to load and $\mathrm{pH}$-dependently release DOX and epirubicin in MCF-7 cells. The toxicity test on the cells indicated that MGC has a lower toxic effect than MG whereas MGC/epirubicin showed a stronger lethal effect on MCF-7 cells. ${ }^{107}$

In a recent work, it was proved that the decoration of GO nanosheets by magnetic iron nanoparticles followed by functionalization by chitosan/sodium alginate ( $\mathrm{CS} / \mathrm{SA})$ leads to the formation of a versatile nanocarrier which can load a high amount of DOX, release it in a low $\mathrm{pH}$ environment and enhance cancer cell killing via photothermal therapy (PTT). ${ }^{108}$ In a similar study, the triple responsive ( $\mathrm{pH}$, thermal and magnetic) $\mathrm{GO}$ nanohydrogel was developed and its DOX release ability was examined in the presence and absence of the magnet. ${ }^{109}$

To overcome the brain-blood barrier (BBB), the functionalized GO/SPION/PLGA loaded by anticancer drug 5-iodo2-deoxyuridine (IUdR) was exposed to an external magnet $(0.5 \mathrm{~T})$ and its elevated accumulation in the glioma-bearing rats was monitored by MRI. The fantastic targeted nanocarrier led to prolong median survival time of the tumor-bearing animals to 38 days. ${ }^{110}$ In order to achieve an efficient chemothermotherapy, the $\mathrm{Fe}_{3} \mathrm{O}_{4}$ nanoparticles were localized into the layered rGo and finally loaded by DOX. Minimum cell survival under hyperthermia induced by a magnetic field occurred in the presence of the nanohybrid. ${ }^{111}$

\section{Graphene-Based Light Responding in DDSs}

As another external stimulus, light can be used to deliver drugs in DDSs for treatment of unhealthy tissues. The DDSs 
based on light stimuli can benefit from user-controllable options like intensity and exposure time to deliver the drug in appropriate time and location. Over the whole electromagnetic spectrum, NIR region because of high transparency into the body as well as low damage to healthy tissues located in the beam path, was served as an excellent stimulator. In this regard, PTT using light absorber nanostructures was extensively explored for the ability of cancer cell ablation at high temperatures. Fortunately, GO nanostructures have a high tendency to absorb NIR radiation and convert it to heat. ${ }^{112}$ On the other hand, photosensitizers loaded on nanocarriers have been utilized for photodynamic therapy (PDT) of cancers. Irradiation of photosensitizers by suitable wavelengths causes the production reactive oxygen species (ROSs) which induce necrotic or apoptotic cells' death. ${ }^{113,114}$

\section{PTT Using Graphene-Based Nanomaterials}

Results of a study showed that peptide targeted mesoporous silica-coated graphene nano-sheet (GSPI) loaded with DOX, could release the drug in acidic $\mathrm{pH}$ and NIR irradiation of 3 -fold higher than the non-irradiation condition. Besides targeting ability, chemo-photothermally cell killing of the nanocomposite was more effective than PTT or chemotherapy alone. ${ }^{115}$ Similarly, the high death of MDA-MB-231 breast cancer cells due to treatment with dual Dox/irinotecan attached to GO NPs as well as NIR exposure has been reported. $^{116}$

In another study, GO and silver were utilized simultaneously for PTT and synthesized DOX loaded GO@Ag targeted with 1,2-distearoyl-sn-glycero-3-phosphoethanolamine (DSPE)-PEG2000-Asn-Gly-Arg (NGR) (GO@AgDOX-NGR). ${ }^{117}$ In addition to imaging contrast-enhancing capability of the nanoplatforms in the animal model, they achieved highest tumor growth inhibition of $83.9 \%$ under irradiation of NIR laser. The same trend in tumor growth was observed using resveratrol (RV)-loaded PEGylated reduced GO (rGO/PEG-RV). ${ }^{118}$

Recently, a novel design for rGO-based DDS has been proposed where rGO-DOX was entrapped in gold nanorods shell-coated vesicle (rGO-AuNRVe) (TEM image, Figure 5A) in order to prevent contact of rGO-DOX with the bio-environment directly. As depicted in Figure $5 \mathrm{~B}$, the rGO-AuNRVe-Dox drug release mechanism into the cell was initiated upon NIR irradiation of it followed by disruption of the vesicle due to heat production and drug detachment from rGO nano-sheets in a $\mathrm{pH}$ dependent manner. Under low $\mathrm{pH}$ and $808 \mathrm{~nm}$ laser irradiation, the nanoplatform showed a unique release profile so that about $70 \%$ of drug is rapidly released over 2 hours. Furthermore, U87MG cells suffered an intensive lethal effect in the presence of rGO-AuNRVe-DOX and laser irradiation simultaneously. ${ }^{119}$ Surprisingly, this multifunctional nanostructure has the ability to improve photoacoustic signals in animal models. ${ }^{119}$ Prodrug Pt(IV) as an analog of cisplatin and a FITC conjugated caspase-3 detector probe, were incorporated on the GO nanosheets surface via amine groups of the PEG chains. In this work, the drug release pattern, PTT effect and potential of apoptosis identification were investigated. They expressed under high levels of GSH-like intracellular environment, $\mathrm{Pt}(\mathrm{IV})$ dihydroxy groups are removed and release $\mathrm{Pt}(\mathrm{II})$. In addition, followed by caspase- 3 activation the florescent quenching of the probe will be halted. However, drug release exhibited GSH concentration dependency whilst PEG-NGO-Pt along with $785 \mathrm{~nm}$ NIR laser irradiation has the highest growth inhibitory effect on 4T1 cells ( $<5 \%$ cell viability for $100 \mu \mathrm{M})$. Remarkably, all PEG-NGO-Pt-treated and laserirradiated tumors of animal models disappeared after 2 days. ${ }^{103}$

A multipurpose nanocarrier using rGO was developed to carry radionuclide ${ }^{131} \mathrm{I}$ attaining nuclear imaging, brachytherapy and PTT concurrently. The rGO-PEG has adequate compatibility with $4 \mathrm{~T} 1$ cells whereas ${ }^{131} \mathrm{I}-\mathrm{rGO}-\mathrm{PEG}$ induced cell death in concentrations that free ${ }^{131} \mathrm{I}$ was unable to kill them. It originates from significant accumulation of ${ }^{131} \mathrm{I}$ in the cells by $\mathrm{rGO}-\mathrm{PEG}$ nanocarrier in comparison to free ${ }^{131} \mathrm{I}$. Moreover, cell counting kit- 8 (CCK8) assay proved 4T1 cells suffer great growth inhabitation after being exposed to ${ }^{131} \mathrm{I}$-rGO-PEG nano-sheet and $808 \mathrm{~nm}$ laser. In contrast to free ${ }^{131} \mathrm{I}$, the ${ }^{131} \mathrm{I}-\mathrm{rGO}-$ PEG was mainly taken up into the tumor rather than the other organs. In vivo tumor growth results showed that tumors were eradicated after 18 days for 4 out of 5 laser rradiated mice and also treated by ${ }^{131}$ I-rGO-PEG. ${ }^{120}$

Moreover for gene therapy, it has been demonstrated the GO functionalized with PEG and PEI can reach to a high plasmid DNA transfection as well as intracellular delivery of small interfering RNA (siRNA) utilizing $808 \mathrm{~nm}$ laser. They reported that heating produced from NIR exposure can elevate permeability of the cell membrane and consequently lead to further delivery of the cargo into the cells. ${ }^{121}$

The synergistic therapeutic effect of a novel light (NIR)- and pH-responsive rGo functionalized by carboxymethyl chitosan and PEG has been reported. Under irradiation of NIR light and low $\mathrm{pH}$, the DOX-oaded 
nanocarrier triggered high-performance PTT and drug release rate. ${ }^{122}$ Similarly, a photothermal agent IR820 (new indocyanine green)-LA (lactobionic acid) was linked to the DOX-GO nanosheets so that in addition to $\mathrm{pH}$ dependent release and photothermal capability, it exhibited fluorescent properties for imaging. ${ }^{123}$

In another work, DOX release pattern from modified $\mathrm{rGO}$ nanosheets was improved under NIR laser irradiation and in an acidic medium. The controllable release manner under laser irradiation can enhance the therapeutic gain by selective light exposure of the target. ${ }^{124}$ In order to load a remarkable amount of indocyanine green (ICG) as a PTT agent, a positive charged magnetic rGO was modified by PEI. As a result, the cancerous cells experience more heat due to irradiation of laser in the presence of the nanocomposite as well as inducing a significant tumor inhibition in the animal model after laser irradiation. Moreover, as a theranostic agent, the nanocomposite can be utilized in MR and thermal imaging modalities. ${ }^{125}$

\section{Graphene-Based Nanostructure-Mediated PDT}

Considering the overexpression of some receptors such as CD44 on the surface of different tumor cells, hyaluronic acid (HA) was attached to GO nano-sheets as a targeting moiety. HA-GO-Ce6 nano-hybrid with a loading content of $115 \%$ extremely quenched $\mathrm{Ce} 6$ fluorescence and reduced the generation of ${ }^{1} \mathrm{O}_{2}$ in comparison with free
Ce6. It was concluded after $\mathrm{Ce} 6$ release from HA-GOCe6 into HeLa cells that it can boost the therapeutic gain of PDT 10 times compared to free Ce6. ${ }^{115}$

Recently, a new nanoplatform was prepared with great potential in synergistic PTT, PDT, and chemotherapy. By Spinach extract (SE) as photosensitizer and contributor in hydrogel formation, rGO and gold nanocages (AuNCs) as NIR absorbers, rGO/AuNCs/SE hydrogel was fabricated to load anticancer drug 5-FU. The 5-FU loaded hydrogel declined the survival rate of HeLa cells to $1.2 \%$ under laser irradiation. $^{126}$

Similarly, methylene blue (MB)-linked GO coated with pluronic copolymer was fabricated to enhance biostability. During protonation of the GO surface and weakening interaction between $\mathrm{MB}$ and $\mathrm{GO}$ due to acidic $\mathrm{pH}$, about $80 \%$ of $\mathrm{MB}$ was released in $\mathrm{pH} 5.0$. Based on the toxicity results, in the form of combined PDT and PTT, the GO-MB nano-photosensitizer persuades HeLa cancer cells to expire more unlike NIH-3T3 normal cells. ${ }^{127}$

It has been shown that a combination of core-shell upconversion NPs (UCNPs), PEGylated GO and photosensitizer medicine of phthalocyanine $(\mathrm{ZnPc})$ forms a nanostructure with luminescence (UCL) emission, PTT and PDT capability. The cytotoxicity test indicated that not only the UCNP-NGO is almost nontoxic for HeLa and KB cells, but UCNP-NGO

A
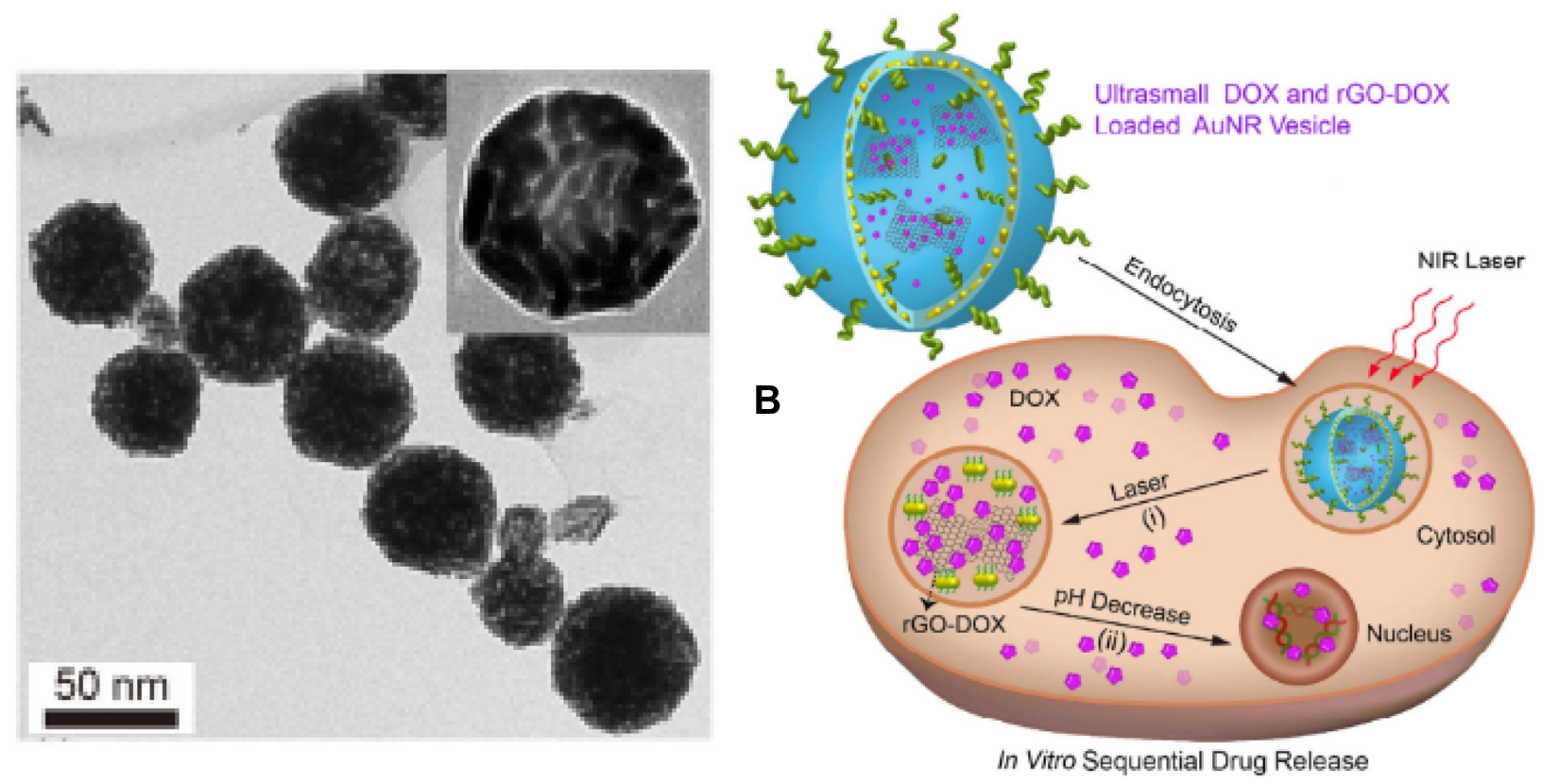

Figure 5 (A) rGO-AuNRVe morphology by TEM, (B) Dox release process from rGO-AuNRVe in the cell (Reproduced with permission).

Note: Copyright @2015. American Chemical Society. Reproduced from Song J, Yang X, Jacobson O, et al. Sequential drug release and enhanced photothermal and photoacoustic effect of hybrid reduced graphene oxide-loaded ultrasmall gold nanorod vesicles for cancer therapy. ACS nano. 2015; 9 (9): $9199-9209 .{ }^{19}$ 
/ZnPc obviously halted the cell growth by heating and singlet oxygen production due to 808 and $630 \mathrm{~nm}$ laser irradiation, respectively. $^{115}$

GO nano-sheets conjugated with the integrin $\alpha v \beta 3$ $\mathrm{mAb}$ were synthesized to transfer pyropheophorbide-a $(\mathrm{PPa})$ photosensitizer into the cell mitochondria as the final target organelle. As expected, uptake test of U87MG (positive) and MCF-7 (negative) cells proved mAb has acted as a proper targeting agent. Moreover, the confocal images clearly showed the PPa-GO-mAb was dominantly accumulated into the mitochondria over $8 \mathrm{~h}$. Once the PPa-GO-mAb nanoplatforms arrived in the mitochondria successfully, a $633 \mathrm{~nm}$ laser can kill cancer cells noticeably. $^{128}$

The difference between GSH levels of intra- and extracellular environment motivated designing of a novel QGD nanocarrier which releases more $\mathrm{Ce} 6$ followed by disulfide link cleavage of GQD-SS-PEG/Ce6 by GSH. They reached drug release percentage $36 \%$ only over 10 hours in contrast to $3 \%$ over 82 hours for $10 \mathrm{mM}$ and $2 \mu \mathrm{M} \mathrm{GSH}$, respectively. The dual stimuli sensitive nanostructure considerably suppressed mice bearing HeLa tumor growth. ${ }^{129}$

In another study, microRNA (miRNA) responding dextran-coated GO nano-colloid was attached with $\mathrm{Ce} 6$ conjugated peptide nucleic acid (PNA). They demonstrated that, in the presence of miR-21, the Ce6-PNA regain its fluorescent and photosensitization ability. Generally, cytotoxicity and tumor growth assay suggested the Ce6-PNA21/Dex-rGO as an appropriate agent for cellular imaging as well as PDT. ${ }^{130}$

In a more recent work, a $\mathrm{pH}$-sensitive link between a photosensitizer agent (Pu18) and GO caused the efficient release of the agent in a weak acidic environment. The authors have claimed that the design can trigger severe damage in the HepG-2 cells via singlet oxygen generation after light irradiation of the photosensitizer agent. ${ }^{124}$ Combining the high tendency of GO to absorb the laser irradiation during PTT, high potential of protoporphyrin IX (PpIX) as a PDT agent and anticancer properties of DOX, led to the synthesis of DOX/GO (PEG-PpIX) nanocomposite. The nano-sized multimodal structure caused a reduction in tumor size of the animal model by a combination of PDT, PTT, and chemotherapy. ${ }^{131}$

Recently, a convenient GQD for using as a photosensitizer was achieved by alternating the number of doped nitrogen atoms. In addition to targeting the cell nucleus the GQDs were functionalized by charge-reversal (3-Aminopropyl) triethoxysilane (APTES) followed by loading DOX. The fluorescent images confirmed the uptake of the nanoplatform into the nucleus where they can significantly harm it by releasing the drug and producing ROS concomitantly. ${ }^{132}$

\section{Graphene in Smart Gene Delivery System}

Smart gene delivery system (SGDS) is simply transferring a foreign DNA to a cell for gene therapy or genetic research. Based on the mechanism of entry of the gene into the cytosol, SGDSs are classified as chemical (like lipids or nanoparticle carriers), biological (like viral or bacterial vectors), or mechanical (like electroporation, microinjection or biolistics). ${ }^{133,134}$

Chemical methods are a more common approach in which genes are delivered via such organic complexes of nucleic acid as lipid, cationic polymer, and calcium phosphate. However, biological methods are based on developing a viral gene delivery, in which the target cells are infected by a specific gene(s). Nevertheless, in the mechanical methods, naked nucleic acids are transferred directly to cells by means of electroporation, microinjection, and phototransfection. ${ }^{135,136}$

As far as the efficiency of a system is concerned, the satisfactory results of a SGDS directly depend on the transport of DNA to selected organs. The advent of nanotechnology is a boom time for different areas of science and industry focus in the 21 st century. ${ }^{57,137}$ Moreover, nanotechnology has an admirable effect in realizing costeffective and efficient diagnostic systems like SGDSs. ${ }^{138}$ With this in mind, novel gene delivery systems for gene therapy can be made with several nanomaterials, including carbon nanotubes (CNTs), ${ }^{139}$ nano-graphene oxide, ${ }^{140}$ nanoscale polymeric materials, as well as peptide and protein-based nanoparticles. ${ }^{141}$

In recent years, the advances of graphene and its various derivatives continue to accelerate as scientists produce a great number of incredible new SGDSs by taking such advantages as flexibility in design, high mechanical strength, low cytotoxicity, high surface area, easy functionalization with targeting ligand, and high intrinsic mobility. ${ }^{142}$ This part highlights the recent advances of graphene and its derivatives like GOs, reduced-GOs (rGOs), and also graphene composites in GDSs and looks forward to the future of graphene-based GDSs.

One of the biggest challenges in using graphene-based nanomaterials for gene delivery is minimizing the side effects of GDS, especially their cytotoxicity. In this regard, 
several studies have modified the graphene derivatives by polymers such as polyethylenimine (PEI), ${ }^{143-146}$ lactosylated chitosan oligosaccharide (LCO), chitosan (CS) ${ }^{147-}$ 149 and polyamidoamine (PAMAM). ${ }^{150,151}$ For instance, the results of Zhang's studies ${ }^{113}$ clearly showed that the use of PEI-conjugated GO to deliver DOX and siRNA does not only lead to improved therapy efficiency but also to patient safety increment. In a similar study, Feng et $\mathrm{al}^{152}$ explored the effects of two molecular weights of PEI $(1.2 \mathrm{kDa}$ and $10 \mathrm{kDa})$ on the cytotoxicity of the PEIGO complex. In a valuable study, Cao et al ${ }^{153}$ highlighted the major role of LCO functionalized graphene oxides in removing the toxicity, and also increasing the loading capacity of FAM-DNA to human hepatic carcinoma cells (QGY-7703).

A GDS was developed to transfer plasmid DNA (pDNA). The electrostatic self-assembly method to prepare folate-conjugated trimethyl chitosan (FTMC/GO) nanoplatforms was applied. Their results showed that pDNA could be loaded into FTMC/GO, in a way that the migration of $31.1 \%$ pDNA could be retarded within 72 hours. They have also observed no cytotoxicity of FTMC/ GO in both Hela cells and A549 cells. Accordingly, FTMC/GO can be noted as being a great candidate for targeted gene delivery. ${ }^{147}$

Liu et al ${ }^{150}$ designed an efficient graphene-based gene vector using graphene-oleate-polyamidoamine (PAMAM) dendrimer hybrids mainly because of their good dispersion and stability in water solutions. They developed this system by means of oleic acid and covalent binding of PAMAM dendrimers. Moreover, they investigated plasmid DNA transfection capacity of the hybrids and the cytotoxicity to HeLa and MG-63 cells. The results of biocompatibility showed that such hybrids are biocompatible to HeLa cells so that up to $100 \mu \mathrm{g} / \mathrm{mL}$ of hybrid, the cellular viability retains about $80 \%$. However, they showed clear cytotoxicity to MG-63 cells at concentrations greater than $20 \mathrm{mg} / \mathrm{mL}$. In optimal condition, the synthesized hybrids enjoy satisfying transfection capacity and biocompatibility.

Based on conjugation of GO and PEI, PAMAM, and polypropylenimine (PPI), three groups of vectors have been fabricated. ${ }^{145}$ They have also used glycine, surface carboxyl group, and spermidine as linkers in their study. Their invaluable results showed that the synthesized vectors generally have lower cytotoxicity in comparison with the intact polymer. Moreover, they evaluated the transfection efficiency of such three groups by a plasmid encoding green fluorescent protein (GFP) using both quantitative and qualitative approaches. The best results were obtained for PEI-based vectors, especially for PEI-GO conjugates with glycine linkers in different carrier to plasmid $(\mathrm{C} / \mathrm{P})$ weight ratios. In addition, these vectors were highly efficient with the best transfection enhancement of 9 fold over that of neat PEI.

\section{Optical Imaging and Theranostics}

The American Cancer Society has predicted that one out of four deaths will be as a result of cancer. Cancer mortality mostly occurs when cancer cells metastasize via the bloodstream. Therefore, early stage detection is a key factor in cancer treatment. Accordingly, recently nanomaterials have been investigated broadly for imaging applications. The right drug for the right patient at the right moment is what nanotechnology-aided theranostics (diagnostic imaging + therapeutic) was born for, which can deliver a noninvasive as well as selective cancer therapy. ${ }^{154}$ PTT/PTD and imaging for diagnosing diseases have been receiving attention due to the fact that unlike most drugs and dyes used for diagnostics they provide no toxicity as well as no side effects like anticancer drugs.

Nano-theranostic therapy has been used through various pathways namely shell-magnetic core star-shaped gold nanoparticles in which the tumor cells were recognized using whole blood sample, ${ }^{155} \mathrm{Fe}_{3} \mathrm{O}_{4} @$ @ Au core/shell nanostars attacking at CD44 receptor-overexpressing cancer cells, ${ }^{156}$ nickel ferrite and carbon targeting melanoma cancer, ${ }^{157}$ or materials such as black phosphorous (BP), covalent organic framework (COF) which are known as graphene-like metal free 2D nanosheets, ${ }^{158}$ and etc. Incorporating graphene in this process has been considered as a novelty of theranostic studies. This led to research on nanocomposites with $\mathrm{Fe}_{2} \mathrm{O}_{3} / \mathrm{Au}$ core-shell nanoparticle-graphene, ${ }^{159}$ PEGylated nanoGO-platine, ${ }^{156}$ nanocomplexes of GO- aptamer, ${ }^{160}$ graphene nanosheetsmagnetic nanoparticles, ${ }^{161}$ acting as theranostic agents. Therefore, graphene-involved theranostic agents have provided a respectable dye-free recognition and treatment procedure.

The unique physical properties of graphene and its derivatives have made them potent nanomaterials to be considered and utilized in several forms of bioimaging systems including: optical imaging, radionuclide-based imaging, MRI (magnetic resonance imaging), Raman imaging and photoacoustic imaging, single-photon emission computed tomography, positron emission tomography, and multimodal imaging. ${ }^{75,162}$ In this part, we only investigate the use of graphene-based nanomaterials in optical imaging which comprises fluorescent imaging (FL), and two-photon fluorescent imaging (TPFL). Non- 
expensive availability, non-ionizing energy, imaging in realtime mode, short-range free-space optical (FSO) communication and multiplexing ability, acceptable photothermal conversion in both first near-infrared window $(650-950 \mathrm{~nm})$ and second near-infrared window (1000-1350) in biological tissue are amongst capabilities of GO- and rGO-based nanomaterials which have resulted in their extensive exploration in the field of bioimaging and theranostics. However, several shortcomings such as low penetration in tissue, high spreading of photons in the visible region by tissue, fluorescent quenching, photobleaching, autofluorescent of background tissue (mainly light-absorbing moieties like water molecules, macronutrients and oxygen-containing groups) have challenged scientists using graphene in theranostic platforms for the last decade begetting future developments in this field. ${ }^{163-166}$ A pH-dependent fluorescence emission of GO flakes in vitro in green $(550 \mathrm{~nm})$ versus red $(630 \mathrm{~nm})$ using $480 \mathrm{~nm}$ laser excitation was explored. Having in mind the acidic environment of cancerous cells (about $\mathrm{pH}=6$ ), the ratio of green to red emission intensities can be used as a $\mathrm{pH}$ sensor for distinguishing healthy cells against tumor cells. ${ }^{15}$ A triple light/enzymeactivated chemotherapy/PDT/PTT theranostics system (HGGNCs/GO-5FU) using hyaluronic acid-glutathione (HG), gold nanoclusters (GNCs), graphene oxide (GO) and the antitumor drug, 5-fluorouracil (5FU) was reported. In this study, it was first shown that HG-GNCs are capable of red fluorescence emission, tumor-targeting function (due to the presence of hyaluronic acid and its ability to target activated CD44 receptor on tumor cells' surfaces) as well as generation of singlet oxygen ${ }^{1} \mathrm{O} 2$ while irradiated by laser. Then the HG-GNCs were combined with 5FU and supported with GO in order to obtain a controlled, targeted fluorescence on/off switch for imaging and synergetic tumor therapy. This was achieved because the fluorescent HG-GNCs served as energy donors and GO as energy acceptor so that the HG-GNCs fluorescence and ${ }^{1} \mathrm{O}_{2}$ generation would be quenched and inhibited by GO. Interestingly, in the presence of hyaluronidase (HAdase), and under laser irradiation, the HG-GNCs and 5FU tended release from GO surface (due to the cleavage of glycosidic bindings) resulting in the restoration of $\mathrm{HG}-\mathrm{GNCs}$ fluorescent and ${ }^{1} \mathrm{O}_{2}$ generation which were combined with photothermal conversion of GO and cancer chemotherapy of 5FU drug. ${ }^{167}$

In another study a red fluorescence, biodegradable, stimuli-responsive nano theranostic platform (GOF-LipFA) was presented based on graphene oxide flakes (GOF) supporting self-assembly liposomes (Lip) loaded with DOX and functionalized with FA (Figure 6A-D). ${ }^{168}$
This theranostic platform was successful both in in vivo diagnosis and treatment of 4T1 breast cancer cells by dualstimuli ( $\mathrm{pH}$ and near-infrared light) of GOF-Lip-FA. The GOF was uniformly attached to the liposome bilayer by electrostatic and hydrophilic interactions of anionic GOF surface and cationic dipalmitoylphosphatidylcholine lipid. The combined chemotherapy and PTT of 4T1 breast tumor regression in mice under $800 \mathrm{~nm}$ laser irradiation and in acidic environments were successfully reported. The drug release was attributed to both protonation (due to the low $\mathrm{pH}$ of cancerous environment) as well as the increased temperature resulting from laser irradiation (Figure $7 \mathrm{~A}-\mathrm{H}){ }^{168}$

Although graphene nanomaterials can bring dye-free theranostics, it has been shown that graphene-based oxides are not intrinsically strong fluorophores, making them needful to other imaging probes or organic dyes to bind with so that they can provide acceptable contrast in bioimaging. ${ }^{16}$ Recently, a GO/ GQD hybrid nanomaterial was prepared as a stable theranostics agent capable of both FL and PTT. In this study, GQDs, as fluorescent agents, were electrostatically bound to GO sheets using PEI as a bridge and also, a fluorescent enhancer (preventing fluorescent quenching of GQDs by GO) leading to GO-PEI-GQDs nanomaterials. This theranostics complex showed great bioimaging and cancer therapy capabilities on MDA-MB-231 cells (breast cancer cells) as well as L292 (healthy murine fibroblast cells) effectively at low dosages and low power density $\left(50 \mu \mathrm{g} / \mathrm{mL}\right.$ and $0.5 \mathrm{~W} . \mathrm{cm}^{-2}$ respectively) of laser (808 nm) exposure. The synergetic effects of PTT and PDT in this research resulted in treatment of cancer cells by both hyperthermia and oxidative stress. ${ }^{169}$

Having an ideal photothermal sensitizer is a crucial tool in ablating destructive cells. Recently scientists have not been able to keep an eye away from graphene in this regard. The most important factor for any material to be used in optical applications (here optical imaging, photothermal treatment) is bandgap because without it emitting light is not possible. This issue is a highlight for graphene since it has no bandgap. What happens in bandgap-less graphene is that via fast electronelectron and electron-photon interactions, carriers relax making it impossible for light emission. ${ }^{170}$ Nevertheless, the good news is graphene in nanosized, $\mathrm{GO}$ and $\mathrm{rGO}$ forms has a bandgap.

Bandgap opening in graphene is a long fascinating story and it has been categorized into two approaches summarized into 1) chemical engineering (chemical functionalization (hydrogenation, fluorination, and other chemical ways); and 2) physical engineering creating defects, doping). ${ }^{171}$ It has to be noted that photoluminescent 
property of graphene-based theranostic platforms is induced by laser to produce heat for malfunction cell destruction.

\section{Angiogenesis and Anti-Angiogenesis Ability of Graphene}

In addition to the high capability of GO nanoplatforms in drug/ gene delivery, some studies paid attention to angiogenesis or anti-angiogenesis properties of them. During angiogenesis new vessels are formed in the primary vessel channels to provide important nutrition and oxygen for rapid proliferative cells and to take away useless biochemical products. As stated in many studies, it plays a key role in wound healing, treatment of cardiovascular diseases as well as tumor growth. ${ }^{172-174}$

99As a booster of angiogenesis for the treatment of cardiovascular diseases, GO nanostructures can act via inducing the intracellular formation of reactive oxygen species (ROS) and reactive nitrogen species (RNS). It has been found that GO and rGo can trigger angiogenesis by a pathway in which ROS could affect the phosphorylation of Akt followed by upregulation of p-eNOS to elevate the intracellular release of NO. This process of angiogenesis can be inverted to antiangiogenesis based on the concentration of GO and rGO as well as the amount of ROS in the cell. ${ }^{175}$ One study has shown that GO/PCL nanoscaffold can induce angiogenesis during sciatic nerve regeneration of rats. According to their result, AKT-eNOS-VEGF signaling was the most probable pathway of the angiogenesis process. ${ }^{176}$ Exploiting the remarkable role of vascular endothelial growth factor (VEGF) in the angiogenesis process, the VEGF-IR800-GO nanotheranostic was developed to monitor and deliver more VEGF into the ischemic tissues concurrently. ${ }^{177}$ Incorporating the nanocomplex of PEI functionalized GO and VEGF gene in a hydrogel caused the effective delivery of the pro-angiogenic gene without any acute toxicity. The myocardial infarction bearing rats were intramyocardially injected by the nanohydrogel. They showed a higher myocardial capillary density and cardiac performance in comparison to other groups. ${ }^{178}$ Recently, as important factors for bone regeneration the capability of GO to stimulate angiogenesis and osteogenesis has been evaluated. The authors revealed GO can induce angiogenesis of HUVACs via VEGF pathway as well as osteogenic differentiation in BMSCs via OSM and NF- $\kappa$ B pathways. ${ }^{179}$ Besides, gelatin-methacryloyl (GelMA) hydrogel loaded by rGO exhibited an acceptable ability of wound healing in endothelial (EA.hy926), Hacat keratinocyte, and 3T3 fibroblast cells. Furthermore, the result of chicken embryo angiogenesis (CEA) assay demonstrated the high potential of the nanocomposite in angiogenesis. ${ }^{180}$

Apart from the angiogenesis properties, the great capacity of GO and its derivatives in carrying and delivering antiangiogenesis drugs has been extensively investigated by many researchers. By administration of $\mathrm{rGO} /$ Dox nanosheets coated by an anti-angiogenic agent (LHT7) into KB tumorbearing mice, they significantly accumulated into the tumor cells compared to $\mathrm{rGO} /$ Dox nanosheets which led to diminishing tumor size after 25 days. ${ }^{181}$ In another study, it has been proven that BSA-GO has a robust binding affinity to VEGF-A $A_{165}$ as the main receptor for triggering angiogenesis. Therefore, the binding can compete with VEGFs and leads to an anti-angiogenesis effect. The treatment of HUVECs by BSA-GO nanosheets caused a notable reduction in proliferation and tube formation of the cells. Additionally, In-vivo results showed the BSA-GO disturbs the angiogenesis process in chick chorioallantoic membrane as well as the formation of blood vessels in rabbit corneal neovascularization. ${ }^{182}$ Similarly, in a more recent study, GO showed an anti-angiogenesis effect on HUVECs through the induction of oxidative stress and the manipulation of some metabolic pathways. ${ }^{183}$ Focusing on the strong correlation between the level of ROS and NF- $\kappa$ B activation, the NF- $\kappa$ B related anti-angiogenesis effect of graphite nanoparticles and graphene oxide nanoplatelets (nGO) was assessed on both p53 mutant and wild type of glioblastoma cancer cells. The treatment of HUVECs co-cultured with p53 wild type of glioblastoma (U87) attenuated their strength for angiogenesis but not for p53 mutant group (U118). The predicted mechanism of anti-angiogenesis of the nanostructure had been attributed to a decrease in the level of ROS and RNS in the cells followed by downregulating of NF- $\mathrm{KB}$ activation which is dependent on p53 status. ${ }^{184}$ Recently, a GO-based nanocarrier was synthesized to deliver a siRNA to silence the VEGF gene. GRcR/VEGF-siRNA downregulated the expression of VEGF in HeLa cells as well as in the animal model which again proves the great potential of GO nanosheets in antiangiogenic approach. ${ }^{185}$

\section{Toxicity and Biocompatibility of Graphene and GO}

Without a doubt, exhaustive investigations using cells and animal models must be conducted in a manner that everything about the biocompatibility and toxicity of graphene and GO is specified, afterward we will be 

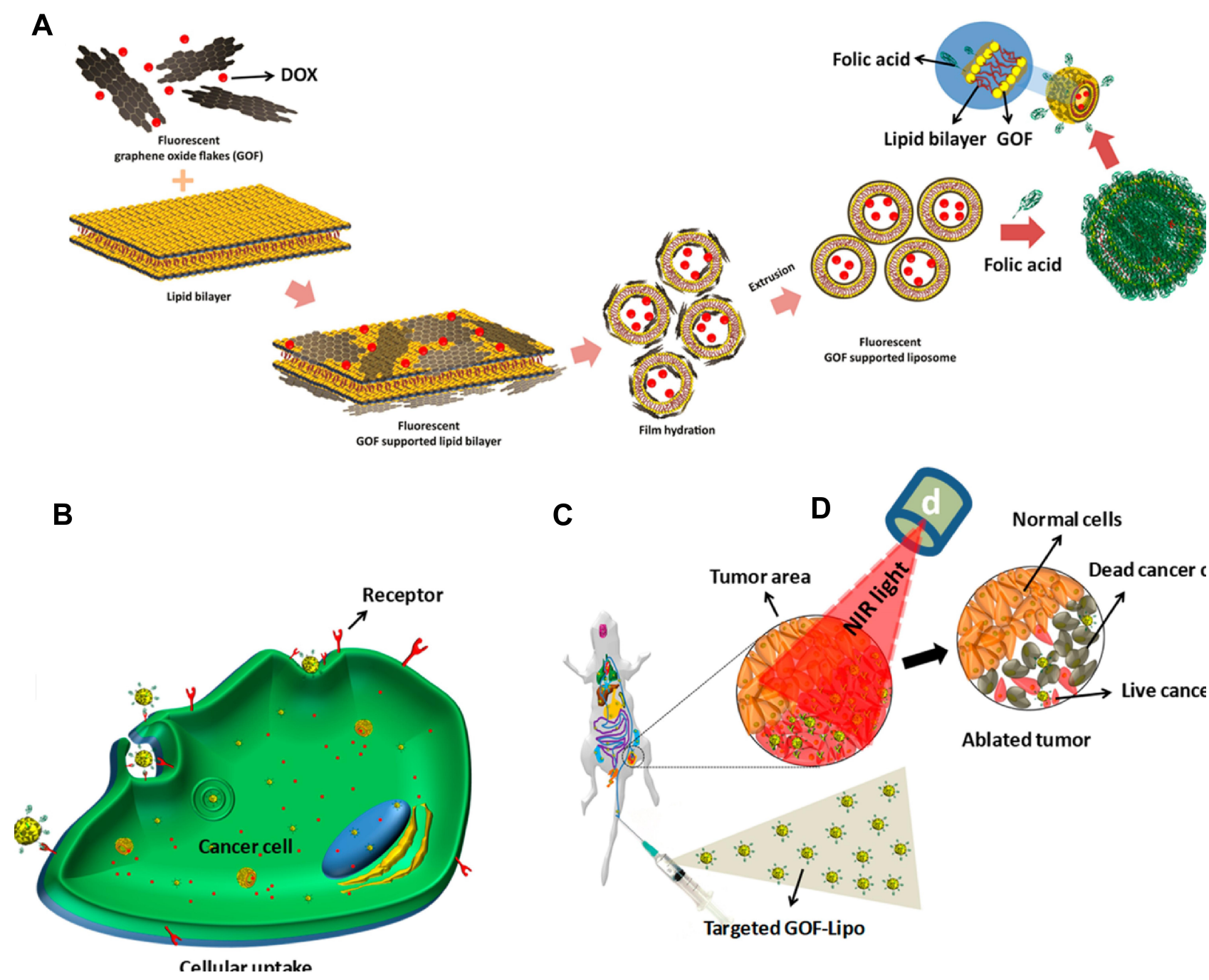

Figure 6 (A) Preparation of Dox loaded GOF-Lip-FA nano theranostic system in a schematic view, (B) Targeted cellular uptake of the GOF-Lip-FA nanohybrid and DOX release in cells body (red particles), (C) In vivo biodistribution of the platform by IV injection and cancer cells uptake, and (D) near-infrared 4TI breast tumor cells death. Note: Copyright (2019. American Chemical Society. Reproduced from Prasad R, Yadav AS, Gorain M, et al. Graphene Oxide Supported Liposomes as Red Emissive Theranostics for Phototriggered Tissue Visualization and Tumor Regression. ACS Applied Bio Materials. 2019; 2 (8): 33।2-3320. ${ }^{168}$

able to talk about their practical clinical applications. Currently, scholars endeavor to assess the toxicity and biocompatibility of GBNs-type structures in order to tackle the problems which arise with the safety of these materials in biological applications.

From the biochemical point of view, the cytotoxicity effect of some synthesized nanomaterials could be expressed via production of ROSs through oxidative stress in treated cells. ${ }^{186}$ There is speculation that oxidative stress is the base of toxicity in GNBs materials considering their analogy to materials that are derived from carbon such as CNTs. ${ }^{187}$ Fibrous-type nanomaterials which include CNTs and GNBs, have been proven to cause adverse health impacts. CNTs and carbon nanofiber have physical similarity to asbestos while exposure to asbestos by inhalation could implement pulmonary diseases such as lung cancer. ${ }^{188}$ Besides, examinations state that the more the dispersibility or solubility of graphene rises, the more its biocompatibility increases, GO sheets encompass plenty of hydrophilic groups such as carboxyl, hydroxyl and epoxy groups on the edges or basal planes, and their hydrophilicity is considerably raised. ${ }^{189}$ Additionally, it has been asserted that the surface functionalization of both graphene and GO with hydrophilic agents, tremendously enhance their biocompatibility. It was reported that gelatinmodified graphene nanosheets were biocompatible while assessed in vitro by MCF-7 cell line, and while loaded with DOX showed high anticancer property. ${ }^{187}$ Other modified types of GO including structures with folic acid conjugation and also sulfonic acid groups modification had proved to be biocompatible. $^{38}$ In other similar researches, PEG as 
Control $15 \mathrm{~min} . \quad 1 \mathrm{~h} \quad 6 \mathrm{~h} \quad 24 \mathrm{~h}$
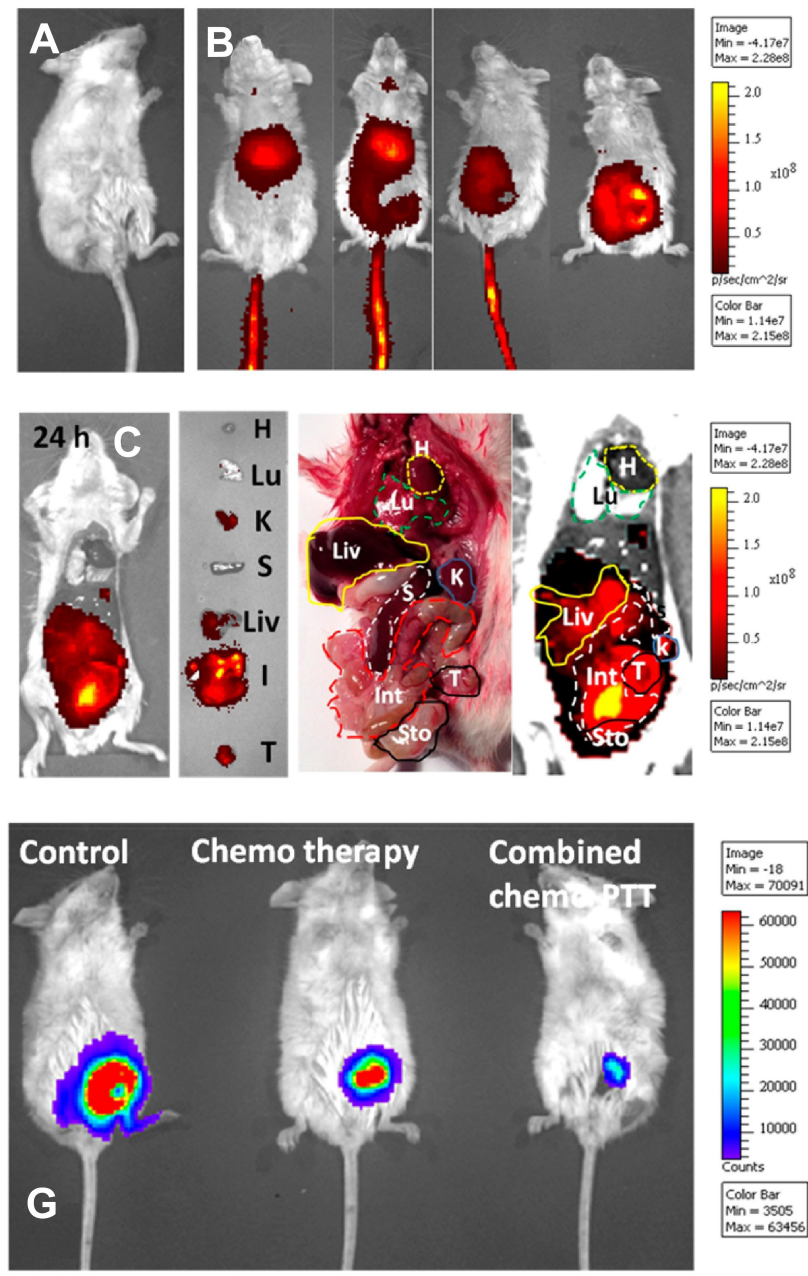

NIR light mediated phototothermal therapy
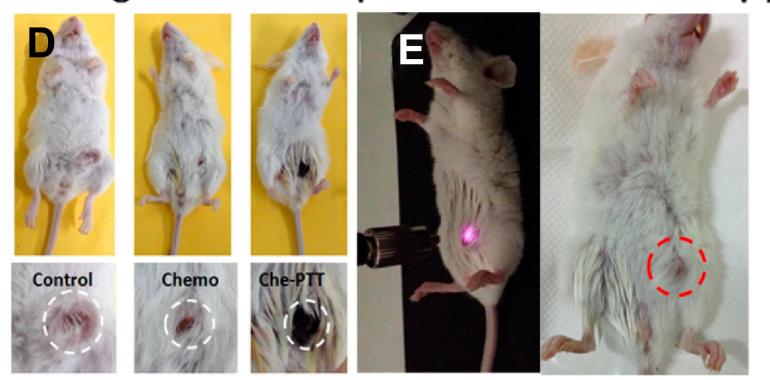

Control

Chemo therapy

Combined chemo-PTT therapy
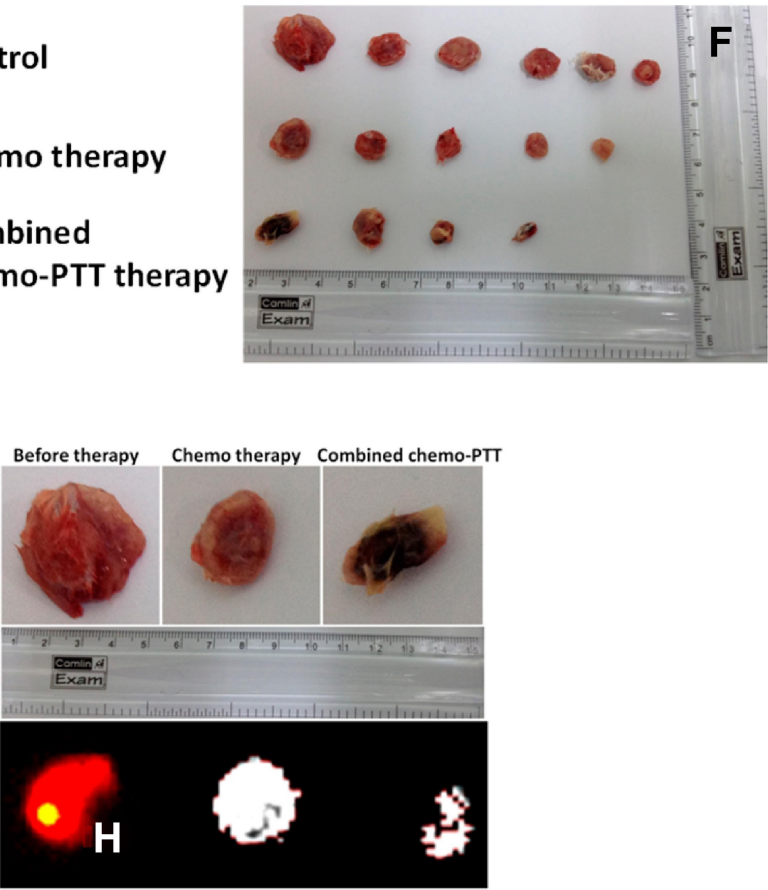

Figure $\mathbf{7}$ In vivo performance of GOF-Lip-FA nano theranostic platform. (A-C) in vivo 4TI cancer diagnosis and distribution of nano theranostic systems by near-infrared fluorescence imaging. (D-E) images of the tumor-bearing animal under laser irradiation and tumor size shrinkage shown in dotted circles. (F) Tumor size after different therapeutic paths. (G) The decrease in tumor size after 21 days. $(\mathbf{H})$ near-infrared fluorescence images of tumor regression after various treatments.

Note: Copyright C2019. American Chemical Society. Reproduced from Prasad R, Yadav AS, Gorain M, et al. Graphene Oxide Supported Liposomes as Red Emissive Theranostics for Phototriggered Tissue Visualization and Tumor Regression. ACS Applied Bio Materials. $2019 ; 2$ (8): 33।2-3320. ${ }^{168}$

a hydrophilic biopolymer has been employed for functionalizing graphene and GO in order to enhance biocompatibility, decrease non-specific binding, and improve in vivo pharmacokinetics for tumor targeting. ${ }^{14}$ Latterly, exploitation of Pluronic F-127 which is another amphiphilic polymer has been reported to functionalize $\mathrm{rGO}^{190}$ and $\mathrm{GO}^{191}$ respectively, both for clinical utilization. The results exhibited that F-127 enhanced stability and solubility of rGO and GO engendering improved biocompatibility and drug performance. In another research, graphene modified by $\mathrm{COOH}^{+}$ion and $\mathrm{COOH}$ were compared in vitro. Better cellular viability was observed for $\mathrm{COOH}^{+}$ implemented graphene. ${ }^{192}$ Silanization of GO nanosheet led to a reduction in cytotoxicity of GO examined on three cell lines namely human dermal fibroblast, murine embryonic fibroblast, and human osteosarcoma. Furthermore, the SiGO showed a lower immune activation of macrophages as well as a less hepatotoxic effect than $\mathrm{GO}$ in the same concentration on the murine model. ${ }^{193}$

With respect to the interaction between distinct forms of graphene and cell membranes, two underlying factors are highly influential. Different cell types as well as morphology of graphene, bringing various materials varying in the number of layers, lateral dimensions, shapes, and chemistry impact on the interaction. To elaborate on this point, it has been disclosed that graphene sheets with a thickness of $10 \mu \mathrm{m}$ could enter the cells by edge-first penetration through the cell membranes and were captured by the lung epithelial cells and macrophages. ${ }^{115}$

Taking all matters into account, in accordance with all of the explorations into biocompatibility, it is unmistakably 
inferred that there is a strong correlation between the two sides; the toxicity of graphene and GO on one side, and their surface functionalization and morphology on the other. Drawing a comparison between functionalized and non-functionalized GBNs determines that the latter are primarily far more toxic. It is crucial to carry out long-term and comprehensive toxicity assessment of the effects of GBNs on people, although such tests have been performed on rodents there is no guarantee that the outcomes can be exploited for humankind as well. ${ }^{194}$ Therefore, prior applying DDSs based on graphene and GO nano-carriers for clinical purposes, numerous in vitro and in vivo toxicity researches are needed.

In terms of biodistribution, multiple pieces of researches have been carried out to evaluate distribution of graphene family materials in living organisms. In 2011, distribution of GO was investigated in mice after intravenous injection. ${ }^{195}$ The results revealed that although several organs had taken up the GO (188Re-GO) during 48 hours, it predominantly accumulated in the lungs, liver, and spleen, with the minimum amount of deposition in the brain, heart, and bone. Over the next few hours, the concentration of GO decreased gradually in most organs, except for the liver and spleen. Another in-vivo research was conducted to investigate the long-term circulation of carboxylated GQDs in treated mice. ${ }^{196}$ The results showed the intravenously administrated nanoparticles mainly are distributed in the liver, lung, spleen, kidney, and tumor sites. Similarly, analogous results of biodistribution were obtained by injecting nanographene sheets into mice. ${ }^{197}$

All in all, when we contemplate the existing available data and information attained in consequence of research works, we become pretty hopeful about the promising entrance of these materials into the world of medical treatment, but still, surely even more endeavors must be made in this regard.

\section{Future Trends}

It is confirmed that over the past few years, the role of graphene and GO in drug/gene delivery has been promoted significantly in view of the unique and unequaled structure and properties ascribed to them. Past researches manifestly demonstrated that the awesome advancements made in this area convey the huge capability of these developing biomaterials to be used in biomedical applications. It is worth noting that the conducted preclinical studies are not sufficient at all and a great deal of preparatory study still needs to be performed. To date, graphene and its derivatives have been profoundly analyzed as a carrier having a large surface area in favor of drug-carrying and particular stress has been laid to characterize their bio- properties in proper cell lines. Notwithstanding, what occurs inside the body after loading the graphene carriers, has not been adequately noticed. The new papers considering the interactions of graphene and cells, plus in vivo effectiveness of these carriers in animals are not sufficient. They have mainly focused on the ability of GBNs to deliver their burden in the body while the attention to their removal is very scarce. In addition, in order to fully appraise their actual capacity, clearance mechanisms, long-term cytotoxicity, tissue biodistribution, and intracellular uptake trends must be studied. Apart from that, having high proficiency in and in-depth knowledge about any type of material based on graphene is essential because each form of graphene-based material possesses its own exclusive properties and surface functionalities, so it should be kept in mind that this does matter in the behavior of the material inside the body. Incidentally, in relation to gene transfection, there is the prospect that its efficiency heightens due to GBNs creation which is at the cutting edge. Unfortunately, the current trend in the studying of therapeutics is not acceptable and should alter as the total concentration is on cancer therapeutics, whereas the therapeutics such as AIDS, neurological disorders, and cardiovascular diseases are not covered in investigations. We are hopful that the present review smooths the path for further and in particular much more comprehensive research works in this field.

Although many efforts have been made to reduce the toxicity of the graphene and graphene-based materials, the use of these compounds is currently associated with high risk. Recently, these materials were put into the list of hazardous agents by The European Scientific Committee on Emerging and Newly Identified Health Risks (SCENIHR). ${ }^{198}$ Unlike simple molecules, when studying a nanomaterial for biomedical research, some critical factors such as shape, size, exposure type and time, agglomeration state and concentration must be considered as a yardstick of bio-safe materials. Talking about graphene family nanomaterials, no unquestionable explanation is available for determining the safety of the materials. However, to clarify the risk-to-benefit balance of graphene-based materials, the main parameters that strongly affect the safety of the materials are necessary to summarize. To put it simply, the interaction between graphene nanomaterials and cells/tissues is the main toxicity issue. Retrospectively, when a graphene-based device is supposed to be as a scaffold in tissue engineering, long time interaction between the device and tissue would be expected. To reduce this concern, efficient synthetic and purification methods must be carefully examined. In significant studies, toxicity is attributed to the contaminants present in the sample. ${ }^{199}$ Along with this 
consideration, rigorous cleaning processes are undeniably needed during top-down strategies for producing graphene materials. For example, during the exfoliation of graphene, amphiphilic substances are usually used that, if not properly cleaned, tissue inflammation might occur. In addition, the morphological characteristics of graphene are very effective in the toxicity of these materials. As an example, although both carbon nanotubes and GO generated using carbon nanomaterials, it has been previously indicated that GO materials have different toxicity for neurons. ${ }^{200}$ As concluded, the difference recognized principally due to the altered shape of the materials. To think about graphene as a bio-grade substance, some other factors such as agglomeration and size must also be considered. Of late, it has been studied that different graphenes in lateral size have different effects on the toxicity of the lung. To be specific, smaller flakes showed less toxicity. ${ }^{47}$ Considering the size of graphene is also important to think about the mechanism by which the material internalizes into a target cell. $^{16}$

Overall, regarding the presented information, it is worthwhile noting that examining a study concerned with graphenebased materials, many factors and properties including size of the material, shape, functionalization, functionalizing groups and percent of these reactions, in-vivo concentration, pharmacokinetic and pharmacodynamics of the materials must carefully be considered.

\section{Disclosure}

The authors report no conflicts of interest in this work.

\section{References}

1. Liu J, Cui L, Losic D. Graphene and graphene oxide as new nanocarriers for drug delivery applications. Acta Biomater. 2013;9 (12):9243-9257. doi:10.1016/j.actbio.2013.08.016

2. Li Z, Fan J, Tong C, et al. A smart drug-delivery nanosystem based on carboxylated graphene quantum dots for tumor-targeted chemotherapy. Nanomedicine. 2019;14(15):2011-2025. doi:10.2217/nnm-2018-0378

3. Novoselov KS, Geim A. The rise of graphene. Nat Mater. 2007;6 (3):183-191. doi:10.1038/nmat1849

4. Pinto AM, Goncalves IC, Magalhães FD. Graphene-based materials biocompatibility: a review. Colloids Surf B Biointerfaces. 2013;111:188-202. doi:10.1016/j.colsurfb.2013.05.022

5. Zangabad PS, Mirkiani S, Shahsavari S, et al. Stimulus-responsive liposomes as smart nanoplatforms for drug delivery applications. Nanotechnol Rev. 2018;7(1):95-122.

6. Farjadian F, Ghasemi A, Gohari O, Roointan A, Karimi M, Hamblin MR. Nanopharmaceuticals and nanomedicines currently on the market: challenges and opportunities. Nanomedicine. 2019;14 (1):93-126. doi:10.2217/nnm-2018-0120

7. Farjadian F, Roointan A, Mohammadi-Samani S, Hosseini M. Mesoporous silica nanoparticles: synthesis, pharmaceutical applications, biodistribution, and biosafety assessment. Chem Eng J. 2019;359:684-705. doi:10.1016/j.cej.2018.11.156
8. Entezar-Almahdi E, Mohammadi-Samani S, Tayebi L, Farjadian F. Recent advances in designing 5-fluorouracil delivery systems: a stepping stone in the safe treatment of colorectal cancer. Int J Nanomedicine. 2020;15:5445. doi:10.2147/IJN. S257700

9. Karimi M, Ghasemi A, Mirkiani S, Basri SMM, Hamblin MR. Carbon Nanotubes in Drug and Gene Delivery. San Rafael, CA: Morgan \& Claypool Publishers; 2017.

10. Fatemeh Farjadian SA, Sadatlu MAA, Mirkiani S, et al. Recent developments in graphene and graphene oxide: properties, synthesis, and modifications: a review chemistryselect. ChemistrySelect. 2020;5(33):10200-10219. doi:10.1002/ slct.202002501

11. A F, Prischepa S, Fedotova J, et al. Electrical conductivity and magnetoresistance in twisted graphene electrochemically decorated with Co particles. Physica E Low Dimens Syst Nanostruct. 2020;117:113790. doi:10.1016/j.physe.2019.113790

12. Jiang J-H, Pi J, Jin H, Cai J-Y. Functional graphene oxide as cancer-targeted drug delivery system to selectively induce oesophageal cancer cell apoptosis. Arti Cells Nanomed Biotechnol. 2018;46(sup3):S297-S307. doi:10.1080/21691401.2018.1492418

13. Muñoz R, Singh DP, Kumar R, Matsuda A. Graphene oxide for drug delivery and cancer therapy. In: Swain SK, Jawaid M, editors. Nanostructured Polymer Composites for Biomedical Applications.. Elsevier; 2019:447-488.

14. Liu Z, Robinson JT, Sun X, Dai H. PEGylated nanographene oxide for delivery of water-insoluble cancer drugs. $\mathrm{J} \mathrm{Am} \mathrm{Chem}$ Soc. 2008;130(33):10876-10877. doi:10.1021/ja803688x

15. Campbell E, Hasan MT, Pho C, Callaghan K, Akkaraju G, Naumov A. Graphene oxide as a multifunctional platform for intracellular delivery, imaging, and cancer sensing. Sci Rep. 2019;9(1):1-9. doi:10.1038/s41598-018-36617-4

16. McCallion C, Burthem J, Rees-Unwin K, Golovanov A, Pluen A. Graphene in therapeutics delivery: problems, solutions and future opportunities. Eur J Pharm Biopharm. 2016;104:235-250. doi:10.1016/j.ejpb.2016.04.015

17. Singh N, Kushwaha P, Gupta A, Prakash O. Recent advances of novel therapeutic agents from botanicals for prevention and therapy of breast cancer: an updated review. Curr Cancer Ther Rev. 2020;16(1):5-18. doi:10.2174/1573394715666181129101502

18. Choi J-S, Joo SH. Recent trends in cyclic peptides as therapeutic agents and biochemical tools. Biomol Ther (Seoul). 2020;28 (1):18. doi:10.4062/biomolther.2019.082

19. Kovalchuk O, Kovalchuk I. Cannabinoids as anticancer therapeutic agents. Cell Cycle. 2020;19(9):961-989. doi:10.1080/ 15384101.2020.1742952

20. Hosseini M, Farjadian F, Makhlouf ASH. Smart stimuliresponsive nano-sized hosts for drug delivery. In: Hosseini, Makhlouf M, Hamdy AS, editors. Industrial Applications for Intelligent Polymers and Coatings.. Springer; 2016:1-26.

21. Abdelhamid HN, Khan MS, Wu H-F. Graphene oxide as a nanocarrier for gramicidin (GOGD) for high antibacterial performance. RSC Adv. 2014;4(91):50035-50046. doi:10.1039/ C4RA07250B

22. Sun X, Liu Z, Welsher K, et al. Nano-graphene oxide for cellular imaging and drug delivery. Nano Res. 2008;1(3):203-212. doi:10.1007/s12274-008-8021-8

23. Goenka S, Sant V, Sant S. Graphene-based nanomaterials for drug delivery and tissue engineering. $J$ Controlled Release. 2014;173:75-88. doi:10.1016/j.jconrel.2013.10.017

24. Lu CH, Yang HH, Zhu CL, Chen X, Chen GN. A graphene platform for sensing biomolecules. Angewandte Chemie. 2009;121(26):4879-4881. doi:10.1002/ange.200901479

25. Ahmadi S, Rabiee N, Bagherzadeh M, et al. Stimulus-responsive sequential release systems for drug and gene delivery. Nano Today. 2020;34:100914. doi:10.1016/j.nantod.2020.100914 
26. Robinson JT, Tabakman SM, Liang Y, et al. Ultrasmall reduced graphene oxide with high near-infrared absorbance for photothermal therapy. J Am Chem Soc. 2011;133(17):6825-6831. doi:10.1021/ja2010175

27. Yang K, Wan J, Zhang S, Tian B, Zhang Y, Liu Z. The influence of surface chemistry and size of nanoscale graphene oxide on photothermal therapy of cancer using ultra-low laser power. Biomaterials. 2012;33(7):2206-2214. doi:10.1016/j. biomaterials.2011.11.064

28. Wang A, Long L, Zhao W, et al. Increased optical nonlinearities of graphene nanohybrids covalently functionalized by axially-coordinated porphyrins. Carbon. 2013;53:327-338. doi:10.1016/j.carbon.2012.11.019

29. Chen Y-W, Chen P-J, Hu S-H, Chen IW, S-Y C. NIR-triggered synergic photo-chemothermal therapy delivered by reduced graphene oxide/carbon/mesoporous silica nanocookies. Adv Funct Mater. 2014;24(4):451-459.

30. Tang $\mathrm{Y}, \mathrm{Hu} \mathrm{H}$, Zhang MG, et al. An aptamer-targeting photoresponsive drug delivery system using "off-on" graphene oxide wrapped mesoporous silica nanoparticles. Nanoscale. 2015;7 (14):6304-6310. doi:10.1039/C4NR07493A

31. Yang K, Feng L, Liu Z. Stimuli responsive drug delivery systems based on nano-graphene for cancer therapy. Adv Drug Deliv Rev. 2016;105(Part):B:228-241. doi:10.1016/j.addr.2016.05.015

32. Yang Y, Zhang YM, Chen Y, Zhao D, Chen JT, Liu Y. Construction of a graphene oxide based noncovalent multiple nanosupramolecular assembly as a scaffold for drug delivery. Chem Eur J. 2012;18 (14):4208-4215. doi:10.1002/chem.201103445

33. Wu J, Y-S W, X-Y Y, et al. Graphene oxide used as a carrier for adriamycin can reverse drug resistance in breast cancer cells. Nanotechnology. 2012;23(35):355101. doi:10.1088/0957-4484/ 23/35/355101

34. Fan X, Jiao G, Gao L, Jin P, Li X. The preparation and drug delivery of a graphene-carbon nanotube-Fe 3 O 4 nanoparticle hybrid. J Mater Chem B. 2013;1(20):2658-2664. doi:10.1039/ c3tb00493g

35. Zhang L, Lu Z, Zhao Q, Huang J, Shen H, Zhang Z. Enhanced chemotherapy efficacy by sequential delivery of siRNA and anticancer drugs using PEI-grafted graphene oxide. Small. 2011;7 (4):460-464. doi:10.1002/smll.201001522

36. Shen $\mathrm{H}$, Liu M, He H, et al. PEGylated graphene oxide-mediated protein delivery for cell function regulation. ACS Appl Mater Interfaces. 2012;4(11):6317-6323. doi:10.1021/am3019367

37. La WG, Park S, Yoon HH, et al. Delivery of a therapeutic protein for bone regeneration from a substrate coated with graphene oxide. Small. 2013;9(23):4051-4060. doi:10.1002/ smll.201300571

38. Zhang L, Xia J, Zhao Q, Liu L, Zhang Z. Functional graphene oxide as a nanocarrier for controlled loading and targeted delivery of mixed anticancer drugs. Small. 2010;6(4):537-544. doi: $10.1002 / \mathrm{smll} .200901680$

39. Öztürk-Atar K, Eroğlu H, Çalış S. Novel advances in targeted drug delivery. J Drug Target. 2018;26(8):633-642. doi:10.1080/ 1061186X.2017.1401076

40. Zhang $\mathrm{M}$, Wu F, Wang W, Shen J, Zhou N, Wu C. Multifunctional nanocomposites for targeted, photothermal, and chemotherapy. Chemi Mater. 2019;31(6):1847-1859. doi:10.1021/acs.chemmater.8b00934

41. De S, Patra K, Ghosh D, et al. Tailoring the efficacy of multifunctional biopolymeric graphene oxide quantum dot-based nanomaterial as nanocargo in cancer therapeutic application. ACS Biomater Sci Eng. 2018;4(2):514-531.

42. Li D, Deng M, Yu Z, et al. Biocompatible and stable GO-coated $\mathrm{Fe} 3 \mathrm{O} 4$ nanocomposite: a robust drug delivery carrier for simultaneous tumor MR imaging and targeted therapy. ACS Biomater Sci Eng. 2018;4(6):2143-2154. doi:10.1021/acsbiomaterials.8b00029
43. Chowdhury AD, Ganganboina AB, Y-C T, H-C C, R-A D. Multifunctional GQDs-Concanavalin A@Fe3O4 nanocomposites for cancer cells detection and targeted drug delivery. Anal Chim Acta. 2018;1027:109-120. doi:10.1016/j.aca.2018.04.029

44. Hussien NA, Işıklan N, Türk M. Aptamer-functionalized magnetic graphene oxide nanocarrier for targeted drug delivery of paclitaxel. Mater Chem Phys. 2018;211:479-488. doi:10.1016/j. matchemphys.2018.03.015

45. Gong P, Ji S, Wang J, et al. Fluorescence-switchable ultrasmall fluorinated graphene oxide with high near-infrared absorption for controlled and targeted drug delivery. Chem Eng J. 2018;348:438-446. doi:10.1016/j.cej.2018.04.193

46. Ratajczak K, Stobiecka M. Ternary interactions and energy transfer between fluorescein isothiocyanate, adenosine triphosphate, and graphene oxide nanocarriers. J Phys Chem B. 2017;121 (28):6822-6830. doi:10.1021/acs.jpcb.7b04295

47. Itatahine A, Mehdi YA, Fizir M, Qi M, Dramou P, He H. Multifunctional carbon nanomateriels for camptothecine low-water soluble anticancer drug delivery. $N$ J Chem. 2018;42 (2):1326-1336. doi:10.1039/C7NJ04609J

48. Deb A, Andrews NG, Raghavan V. Natural polymer functionalized graphene oxide for co-delivery of anticancer drugs: in-vitro and in-vivo. Int $J$ Biol Macromol. 2018;113:515-525. doi:10.1016/j.ijbiomac.2018.02.153

49. Zare-Zardini H, Taheri-Kafrani A, Amiri A, Bordbar AK. New generation of drug delivery systems based on ginsenoside Rh2-, Lysine- and Arginine-treated highly porous graphene for improving anticancer activity. Sci Rep. 2018;8(1):1-15. doi:10.1038/ s41598-017-18938-y

50. Javanbakht S, Namazi H. Doxorubicin loaded carboxymethyl cellulose/graphene quantum dot nanocomposite hydrogel films as a potential anticancer drug delivery system. Mater Sci Eng C. 2018;87:50-59. doi:10.1016/j.msec.2018.02.010

51. Li Y, Gao X, Yu Z, et al. Reversing multidrug resistance by multiplexed gene silencing for enhanced breast cancer chemotherapy. ACS Appl Mater Interfaces. 2018;10 (18):15461-15466. doi:10.1021/acsami.8b02800

52. Xie M, Zhang F, Liu L, et al. Surface modification of graphene oxide nanosheets by protamine sulfate/sodium alginate for anti-cancer drug delivery application. Appl Surf Sci. 2018;440:853-860. doi:10.1016/j.apsusc.2018.01.175

53. Puvirajesinghe TM, Zhi Z, Craster R, Guenneau S. Tailoring drug release rates in hydrogel-based therapeutic delivery applications using graphene oxide. J R Soc Interface. 2018;15(139):20170949. doi:10.1098/rsif.2017.0949

54. Rao Z, Ge H, Liu L, et al. Carboxymethyl cellulose modified graphene oxide as pH-sensitive drug delivery system. Int J Biol Macromol. 2018;107:1184-1192. doi:10.1016/j.ijbiomac.2017.09.096

55. Deb A, Vimala R. Camptothecin loaded graphene oxide nanoparticle functionalized with polyethylene glycol and folic acid for anticancer drug delivery. J Drug Deliv Sci Technol. 2018;43 (October2017):333-342. doi:10.1016/j.jddst.2017.10.025

56. de Sousa M, Visani de Luna LA, Fonseca LC, Giorgio S, Alves OL. Folic-acid-functionalized graphene oxide nanocarrier: synthetic approaches, characterization, drug delivery study, and antitumor screening. ACS Appl Nano Mater. 2018;1(2):922-932. doi:10.1021/acsanm.7b00324

57. Karimi M, Eslami M, Sahandi-Zangabad P, et al. pH-Sensitive stimulus-responsive nanocarriers for targeted delivery of therapeutic agents. Wiley Interdiscip Rev Nanomed Nanobiotechnol. 2016;8(5):696-716.

58. Roointan A, Farzanfar J, Mohammadi-Samani S, BehzadBehbahani A, Farjadian F. Smart pH responsive drug delivery system based on poly (HEMA-co-DMAEMA) nanohydrogel. Int $J$ Pharm. 2018;552(1-2):301-311. doi:10.1016/j.ijpharm. 2018.10.001 
59. Mozafari N, Farjadian F, Samani SM, Azadi S, Azadi A. Simvastatinchitosan-citicoline conjugates nanoparticles as the co-delivery system in Alzheimer susceptible patients. Int $J$ Biol Macromol. 2020;156:1396-1407. doi:10.1016/j.ijbiomac.2019.11.180

60. Kavitha T, Abdi SIH, Park S-Y. pH-sensitive nanocargo based on smart polymer functionalized graphene oxide for site-specific drug delivery. Phys Chem Chem Phys. 2013;15(14):5176-5185. doi:10.1039/c3cp00008g

61. Wang C, Chen B, Zou M, Cheng G. Cyclic RGD-modified chitosan/graphene oxide polymers for drug delivery and cellular imaging. Colloids Surf B Biointerfaces. 2014;122:332-340. doi:10.1016/j.colsurfb.2014.07.018

62. Feng L, Li K, Shi X, Gao M, Liu J, Liu Z. Smart pH-responsive nanocarriers based on nano-graphene oxide for combined chemo-and photothermal therapy overcoming drug resistance. Adv Healthcare Mater. 2014;3(8):1261-1271. doi:10.1002/adhm.201300549

63. Jiang T, Sun $\mathrm{W}$, Zhu Q, et al. Furin-mediated sequential delivery of anticancer cytokine and small-molecule drug shuttled by graphene. Adv Mater. 2015;27(6):1021-1028. doi:10.1002/ adma.201404498

64. Lv O, Tao Y, Qin Y, et al. Highly fluorescent and morphology-controllable graphene quantum dots-chitosan hybrid xerogels for in vivo imaging and $\mathrm{pH}$-sensitive drug carrier. Mater Sci Eng C. 2016;67:478-485. doi:10.1016/j.msec.2016.05.031

65. Qiu J, Zhang R, Li J, et al. Fluorescent graphene quantum dots as traceable, $\mathrm{pH}$-sensitive drug delivery systems. Int J Nanomedicine. 2015;10:6709.

66. Huang Y-S, Lu Y-J, Chen J-P. Magnetic graphene oxide as a carrier for targeted delivery of chemotherapy drugs in cancer therapy. J Magn Magn Mater. 2016.

67. Tyagi N, Attia NF, Geckeler KE. Exfoliated graphene nanosheets: pH-sensitive drug carrier and anti-cancer activity. J Colloid Interface Sci. 2017;498:364-377. doi:10.1016/j.jcis.2017.03.057

68. Javanbakht S, Shaabani A. Encapsulation of graphene quantum dot-crosslinked chitosan by carboxymethylcellulose hydrogel beads as a $\mathrm{pH}$-responsive bio-nanocomposite for the oral delivery agent. Int J Biol Macromol. 2019;123:389-397. doi:10.1016/j. ijbiomac.2018.11.118

69. Dong J, Wang K, Sun L, et al. Application of graphene quantum dots for simultaneous fluorescence imaging and tumor-targeted drug delivery. Sens Actuators B Chem. 2018;256:616-623. doi:10.1016/j.snb.2017.09.200

70. Sumathra M, Sadasivuni KK, Kumar SS, Rajan M. Cisplatin-Loaded graphene oxide/chitosan/hydroxyapatite composite as a promising tool for osteosarcoma-affected bone regeneration. ACS Omega. 2018;3(11):14620-14633. doi:10.1021/acsomega.8b02090

71. Vinothini K, Rajendran NK, Rajan M, Ramu A, Marraiki N, Elgorban AM. A magnetic nanoparticle functionalized reduced graphene oxide-based drug carrier system for a chemo-photodynamic cancer therapy. $N$ J Chem. 2020;44 (14):5265-5277. doi:10.1039/D0NJ00049C

72. Vinothini K, Rajendran NK, Ramu A, Elumalai N, Rajan M. Folate receptor targeted delivery of paclitaxel to breast cancer cells via folic acid conjugated graphene oxide grafted methyl acrylate nanocarrier. Biomedicine Pharmacother. 2019;110:906-917. doi:10.1016/j. biopha.2018.12.008

73. Vinothini K, Rajendran NK, Munusamy MA, Alarfaj AA, Rajan M. Development of biotin molecule targeted cancer cell drug delivery of doxorubicin loaded $\kappa$-carrageenan grafted graphene oxide nanocarrier. Mater Sci Eng C. 2019;100:676-687. doi:10.1016/j.msec.2019.03.011

74. Prabakaran S, Jeyaraj M, Nagaraj A, Sadasivuni KK, Rajan M. Polymethyl methacrylate-ovalbumin@ graphene oxide drug carrier system for high anti-proliferative cancer drug delivery. Applied Nanosci. 2019;9(7):1487-1500. doi:10.1007/s13204-019-00950-5
75. Luo Y, Tang Y, Liu T, et al. Engineering graphene oxide with ultrasmall SPIONs and smart drug release for cancer theranostics. Chem Commun. 2019;55(13):1963-1966. doi:10.1039/C8CC0 9185D

76. Kazempour M, Namazi H, Akbarzadeh A, Kabiri R. Synthesis and characterization of PEG-functionalized graphene oxide as an effective pH-sensitive drug carrier. Artif Cells Nanomed Biotechnol. 2019;47 (1):90-94. doi:10.1080/21691401.2018.1543196

77. Kundu A, Nandi S, Das P, Nandi AK. Fluorescent graphene oxide via polymer grafting: an efficient nanocarrier for both hydrophilic and hydrophobic drugs. ACS Appl Mater Interfaces. 2015;7 (6):3512-3523. doi:10.1021/am507110r

78. Onuki Y, Bhardwaj U, Papadimitrakopoulos F, Burgess DJ. A Review of the Biocompatibility of Implantable Devices: Current Challenges to Overcome Foreign Body Response. SAGE Publications; 2008.

79. Romberg B, Hennink WE, Storm G. Sheddable coatings for long-circulating nanoparticles. Pharm Res. 2008;25(1):55-71. doi:10.1007/s11095-007-9348-7

80. Meng F, Hennink WE, Zhong Z. Reduction-sensitive polymers and bioconjugates for biomedical applications. Biomaterials. 2009;30(12):2180-2198. doi:10.1016/j.biomaterials.2009.01.026

81. Wen H, Dong C, Dong H, et al. Engineered redox-responsive PEG detachment mechanism in PEGylated nano-graphene oxide for intracellular drug delivery. Small. 2012;8(5):760-769. doi:10.1002/smll.201101613

82. Chen H, Wang Z, Zong S, et al. SERS-fluorescence monitored drug release of a redox-responsive nanocarrier based on graphene oxide in tumor cells. ACS Appl Mater Interfaces. 2014;6 (20):17526-17533. doi:10.1021/am505160v

83. Zhao X, Yang L, Li X, et al. Functionalized graphene oxide nanoparticles for cancer cell-specific delivery of antitumor drug. Bioconjug Chem. 2015;26(1):128-136. doi:10.1021/ bc5005137

84. Al-Nahain A, Lee SY, In I, Lee KD, Park SY. Triggered pH/redox responsive release of doxorubicin from prepared highly stable graphene with thiol grafted pluronic. Int J Pharm. 2013;450 (1):208-217. doi:10.1016/j.ijpharm.2013.04.053

85. Cho Y, Choi Y. Graphene oxide-photosensitizer conjugate as a redox-responsive theranostic agent. Chem Commun. 2012;48 (79):9912-9914. doi:10.1039/c2cc35197h

86. Yin $\mathrm{T}$, Liu J, Zhao $\mathrm{Z}$, et al. Redox sensitive hyaluronic acid-decorated graphene oxide for photothermally controlled tumor-cytoplasm-selective rapid drug delivery. Adv Funct Mater. 2017;27(14):1604620. doi:10.1002/adfm.201604620

87. Kim DJ, Kim J, Lee HL, et al. Redox-responsive nanocomposites composed of graphene oxide and chlorin e6 for photodynamic treatment of cholangiocarcinoma. Bull Korean Chem Soc. 2018;39(9):1073-1082. doi:10.1002/bkcs.11552

88. Liu J, Zhang D, Lian S, et al. Redox-responsive hyaluronic acid-functionalized graphene oxide nanosheets for targeted delivery of water-insoluble cancer drugs. Int $J$ Nanomedicine. 2018;13:7457. doi:10.2147/IJN.S173889

89. Ma N, Song A, Li Z, Luan Y. Redox-sensitive prodrug molecules meet graphene oxide: an efficient graphene oxide-based nanovehicle toward cancer therapy. ACS Biomater Sci Eng. 2019;5 (3):1384-1391. doi:10.1021/acsbiomaterials.9b00114

90. Farjadian F, Rezaeifard S, Naeimi M, et al. Temperature and pH-responsive nano-hydrogel drug delivery system based on lysine-modified poly (vinylcaprolactam). Int J Nanomedicine. 2019;14:6901. doi:10.2147/IJN.S214467

91. Farjadian F, Ghasemi S, Andami Z, Tamami B. Thermo-responsive nanocarrier based on poly ( $\mathrm{N}$-isopropylacrylamide) serving as a smart doxorubicin delivery system. Iranian Polymer J. 2020;29(3):197-207. doi:10.1007/s13726-020-00785-w 
92. Zhu S, Li J, Chen Y, et al. Grafting of graphene oxide with stimuli-responsive polymers by using ATRP for drug release. J Nanoparticle Res. 2012;14(9):1-11. doi:10.1007/s11051-0121132-X

93. Wang H, Sun D, Zhao N, et al. Thermo-sensitive graphene oxidepolymer nanoparticle hybrids: synthesis, characterization, biocompatibility and drug delivery. J Mater Chem B. 2014;2 (10):1362-1370.

94. Pan Y, Bao H, Sahoo NG, Wu T, Water-Soluble Poly LL. (Nisopropylacrylamide)-graphene sheets synthesized via click chemistry for drug delivery. Adv Funct Mater. 2011;21 (14):2754-2763. doi:10.1002/adfm.201100078

95. Bardajee GR, Hooshyar Z, Farsi M, Mobini A, Sang G. Synthesis of a novel thermo/pH sensitive nanogel based on salep modified graphene oxide for drug release. Mater Sci Eng C. 2016.

96. Wang C, Mallela J, Garapati USet al., A chitosan-modified graphene nanogel for noninvasive controlled drug release. Nanomedicine. 2013;9(7):903-911. doi:10.1016/j.nano.2013.01.003

97. Wang P, Chen S, Cao Z, Wang G, Light- NIR. Temperature-, pH-, and redox-responsive polymer-modified reduced graphene oxide/ mesoporous silica sandwich-like nanocomposites for controlled release. ACS Appl Mater Interfaces. 2017;9(34):29055-29062. doi:10.1021/acsami.7b07468

98. Khoee S, Karimi MR. Dual-drug loaded Janus graphene oxide-based thermoresponsive nanoparticles for targeted therapy. Polymer. 2018;142:80-98. doi:10.1016/j.polymer.2018.03.022

99. Havanur S, Batish I, Cheruku SP, Gourishetti K, Kumar PEJ. N. Poly(N,N-diethyl acrylamide)/functionalized graphene quantum dots hydrogels loaded with doxorubicin as a nano-drug carrier for metastatic lung cancer in mice. Mater Sci Eng C. 2019;105:110094. doi:10.1016/j.msec.2019.110094

100. Farjadian F, Ghasemi S, Mohammadi-Samani S. Hydroxylmodified magnetite nanoparticles as novel carrier for delivery of methotrexate. Int J Pharm. 2016;504(1-2):110-116. doi:10.1016/ j.ijpharm.2016.03.022

101. Yang X, Wang Y, Huang X, et al. Multi-functionalized graphene oxide based anticancer drug-carrier with dual-targeting function and pH-sensitivity. J Mater Chem. 2011;21(10):3448-3454. doi:10.1039/C0JM02494E

102. Wang Z, Zhou C, Xia J, et al. Fabrication and characterization of a triple functionalization of graphene oxide with $\mathrm{Fe} 3 \mathrm{O}$, folic acid and doxorubicin as dual-targeted drug nanocarrier. Colloids Surf B Biointerfaces. 2013;106:60-65. doi:10.1016/j.colsurfb. 2013.01.032

103. Swain AK, Pradhan L, Bahadur D. Polymer stabilized Fe3O4-graphene as an amphiphilic drug carrier for thermo-chemotherapy of cancer. ACS Appl Mater Interfaces. 2015;7(15):8013-8022. doi:10.1021/acsami.5b02536

104. Ma X, Tao H, Yang K, et al. A functionalized graphene oxide-iron oxide nanocomposite for magnetically targeted drug delivery, photothermal therapy, and magnetic resonance imaging. Nano Res. 2012;5(3):199-212. doi:10.1007/s12274-012-0200-y

105. Wang C, Ravi S, Garapati USet al., Multifunctional chitosan magnetic-graphene (CMG) nanoparticles: a theranostic platform for tumor-targeted co-delivery of drugs, genes and MRI contrast agents. J Mater Chem B. 2013;1(35):4396-4405. doi:10.1039/ c3tb20452a

106. Guo L, Shi H, Wu H, et al. Prostate cancer targeted multifunctionalized graphene oxide for magnetic resonance imaging and drug delivery. Carbon. 2016;107:87-99. doi:10.1016/j.carbon.2016. 05.054

107. Wang $\mathrm{C}$, Li B, Niu W, et al. $\beta$-Cyclodextrin modified graphene oxide-magnetic nanocomposite for targeted delivery and $\mathrm{pH}$ sensitive release of stereoisomeric anti-cancer drugs. RSC Adv. 2015;5(108):89299-89308. doi:10.1039/C5RA13082D
108. Xie M, Zhang F, Peng H, et al. Layer-by-layer modification of magnetic graphene oxide by chitosan and sodium alginate with enhanced dispersibility for targeted drug delivery and photothermal therapy. Colloids Surf B Biointerfaces. 2019;176:462-470. doi:10.1016/j.colsurfb.2019.01.028

109. Bardajee GR, Hooshyar Z. Thermo/pH/magnetic-triple sensitive poly(N-isopropylacrylamide-co-2-dimethylaminoethyl) methacrylate)/sodium alginate modified magnetic graphene oxide nanogel for anticancer drug delivery. Polymer Bull. 2018;75 (12):5403-5419. doi:10.1007/s00289-018-2329-7

110. Shirvalilou S, Khoei S, Khoee S, Raoufi NJ, Karimi MR, ShakeriZadeh A. Development of a magnetic nano-graphene oxide carrier for improved glioma-targeted drug delivery and imaging: in vitro and in vivo evaluations. Chem Biol Interact. 2018;295:97-108. doi:10.1016/j.cbi.2018.08.027

111. Gupta J, Prakash A, Jaiswal MK, Agarrwal A, Bahadur D. Superparamagnetic iron oxide-reduced graphene oxide nanohybrid-a vehicle for targeted drug delivery and hyperthermia treatment of cancer. J Magn Magn Mater. 2018;448:332-338. doi:10.1016/j.jmmm.2017.05.084

112. Bao Z, Liu X, Liu Y, Liu H, Zhao K. Near-infrared light-responsive inorganic nanomaterials for photothermal therapy. Asian J Pharm Sci. 2016;11(3):349-364. doi:10.1016/j.ajps.2015.11.123

113. Tian B, Wang C, Zhang S, Feng L, Liu Z. Photothermally enhanced photodynamic therapy delivered by nano-graphene oxide. ACS Nano. 2011;5(9):7000-7009. doi:10.1021/nn201560b

114. Zhou L, Jiang H, Wei S, Ge X, Zhou J, Shen J. High-efficiency loading of hypocrellin $\mathrm{B}$ on graphene oxide for photodynamic therapy. Carbon. 2012;50(15):5594-5604. doi:10.1016/j. carbon.2012.08.013

115. Wang Y, Wang H, Liu D, Song S, Wang X, Zhang H. Graphene oxide covalently grafted upconversion nanoparticles for combined NIR mediated imaging and photothermal/photodynamic cancer therapy. Biomaterials. 2013;34(31):7715-7724. doi:10. 1016/j.biomaterials.2013.06.045

116. Tran TH, Nguyen HT, Pham TT, et al. Development of a graphene oxide nanocarrier for dual-drug chemo-phototherapy to overcome drug resistance in cancer. ACS Appl Mater Interfaces. 2015;7 (51):28647-28655. doi:10.1021/acsami.5b10426

117. Shi J, Wang L, Zhang J, et al. A tumor-targeting near-infrared laser-triggered drug delivery system based on GO@ Ag nanoparticles for chemo-photothermal therapy and X-ray imaging. Biomaterials. 2014;35(22):5847-5861. doi:10.1016/j. biomaterials.2014.03.042

118. Chen J, Liu H, Zhao C, et al. One-step reduction and PEGylation of graphene oxide for photothermally controlled drug delivery. Biomaterials. 2014;35(18):4986-4995. doi:10.1016/j.biomaterials. 2014.02.032

119. Song J, Yang X, Jacobson O, et al. Sequential drug release and enhanced photothermal and photoacoustic effect of hybrid reduced graphene oxide-loaded ultrasmall gold nanorod vesicles for cancer therapy. ACS Nano. 2015;9(9):9199-9209. doi:10. 1021/acsnano.5b03804

120. Chen L, Zhong X, Yi X, et al. Radionuclide 131 I labeled reduced graphene oxide for nuclear imaging guided combined radio-and photothermal therapy of cancer. Biomaterials. 2015;66:21-28. doi:10.1016/j.biomaterials.2015.06.043

121. Feng L, Yang X, Shi X, et al. Polyethylene glycol and polyethylenimine dual-functionalized nano-graphene oxide for photothermally enhanced gene delivery. Small. 2013;9(11):1989-1997. doi:10.1002/smll.201202538

122. Liu W, Zhang X, Zhou L, Shang L, Su Z. Reduced graphene oxide (rGO) hybridized hydrogel as a near-infrared (NIR)/pH dual-responsive platform for combined chemo-photothermal therapy. J Colloid Interface Sci. 2019;536:160-170. doi:10.1016/ j.jcis.2018.10.050 
123. Huang C, Hu X, Hou Z, Ji J, Li Z, Luan Y. Tailored graphene oxide-doxorubicin nanovehicles via near-infrared dye-lactobionic acid conjugates for chemo-photothermal therapy. $J$ Colloid Interface Sci. 2019;545:172-183. doi:10.1016/j.jcis.2019.03.019

124. Zhang Y, Zhang H, Wang Z, Jin Y. pH-Sensitive graphene oxide conjugate purpurin-18 methyl ester photosensitizer nanocomplex in photodynamic therapy. $N J$ Chem. 2018;42(16):13272-13284. doi:10.1039/C8NJ00439K

125. Du B, Liu J, Ding Get al., Positively charged graphene/Fe3O4/ polyethylenimine with enhanced drug loading and cellular uptake for magnetic resonance imaging and magnet-responsive cancer therapy. Nano Res. 2017;10(7):2280-2295. doi:10.1007/s12274016-1418-x

126. Chang G, Li S, Huang F, Zhang X, Shen Y, Xie A. Multifunctional reduced graphene oxide hydrogel as drug carrier for localized and synergic photothermal/photodynamics/chemo therapy. J Mater Sci Technol. 2016;32(8):753-762. doi:10.1016/ j.jmst.2016.06.014

127. Sahu A, Choi WI, Lee JH, Tae G. Graphene oxide mediated delivery of methylene blue for combined photodynamic and photothermal therapy. Biomaterials. 2013;34(26):6239-6248. doi:10.1016/j.biomaterials.2013.04.066

128. Wei Y, Zhou F, Zhang D, Chen Q, Xing D. A graphene oxide based smart drug delivery system for tumor mitochondria-targeting photodynamic therapy. Nanoscale. 2016;8(6):3530-3538. doi:10.1039/C5NR07785K

129. Li Y, Wu Z, Du D, Dong H, Shi D, Li Y. A graphene quantum dot (GQD) nanosystem with redox-triggered cleavable PEG shell facilitating selective activation of the photosensitiser for photodynamic therapy. RSC $A d v .2016 ; 6(8): 6516-6522$. doi:10.1039/ C5RA23622C

130. Lee JS, Kim S, Na HK, Min DH. MicroRNA-responsive drug release system for selective fluorescence imaging and photodynamic therapy in vivo. Adv Healthcare Mater. 2016;5 (18):2386-2395. doi:10.1002/adhm.201600328

131. Su T, Cheng F, Yan J, et al. Hierarchical nanocomposites of graphene oxide and PEGylated protoporphyrin as carriers to load doxorubicin hydrochloride for trimodal synergistic therapy. J Mater Chem B. 2018;6(28):4687-4696. doi:10.1039/C8TB00733K

132. Ju J, Regmi S, Fu A, Lim S, Liu Q. Graphene quantum dot based charge-reversal nanomaterial for nucleus-targeted drug delivery and efficiency controllable photodynamic therapy. J Biophoton. 2019;12(6):e201800367. doi:10.1002/jbio.201800367

133. Pack DW, Hoffman AS, Pun S, Stayton PS. Design and development of polymers for gene delivery. Nat Rev Drug Discov. 2005;4 (7):581. doi: $10.1038 / \mathrm{nrd} 1775$

134. Panyam J, Labhasetwar V. Biodegradable nanoparticles for drug and gene delivery to cells and tissue. Adv Drug Deliv Rev. 2003;55(3):329-347. doi:10.1016/S0169-409X(02)00228-4

135. Xiang Y, Oo NNL, Lee JP, Li Z, Loh XJ. Recent development of synthetic nonviral systems for sustained gene delivery. Drug Discov Today. 2017;22(9):1318-1335. doi:10.1016/j.drudis.2017. 04.001

136. Midoux P, Pichon C, Yaouanc JJ, Jaffrès PA. Chemical vectors for gene delivery: a current review on polymers, peptides and lipids containing histidine or imidazole as nucleic acids carriers. $\mathrm{Br}$ $J \quad$ Pharmacol. 2009;157(2):166-178. doi:10.1111/j.14765381.2009.00288.x

137. Farjadian F, Moghoofei M, Mirkiani S, et al. Bacterial components as naturally inspired nano-carriers for drug/gene delivery and immunization: set the bugs to work? Biotechnol Adv. 2018;36 (4):968-985. doi:10.1016/j.biotechadv.2018.02.016

138. Wong JK, Mohseni R, Hamidieh AA, MacLaren RE, Habib N, Seifalian AM. Will nanotechnology bring new hope for gene delivery? Trends Biotechnol. 2017;35(5):434-451. doi:10.1016/j. tibtech.2016.12.009
139. Karimi M, Solati N, Ghasemi A, et al. Carbon nanotubes part II: a remarkable carrier for drug and gene delivery. Expert Opin Drug Deliv. 2015;12(7):1089-1105. doi:10.1517/17425247.2015.1004309

140. Chandrasekhar P. Medical and pharmaceutical applications of graphene. In: Dalton, editor. Conducting Polymers, Fundamentals and Applications. Springer; 2018:149-150.

141. Zhi F, Dong H, Jia X, et al. Functionalized graphene oxide mediated adriamycin delivery and miR-21 gene silencing to overcome tumor multidrug resistance in vitro. PLoS One. 2013;8(3): e60034. doi:10.1371/journal.pone.0060034

142. Goncalves G, Marques P, Vila M. Graphene-Based Materials in Health and Environment. Springer; 2017.

143. Jian H-J, Wu R-S, Lin T-Y, et al. Super-cationic carbon quantum dots synthesized from spermidine as an eye drop formulation for topical treatment of bacterial keratitis. ACS Nano. 2017;11 (7):6703-6716. doi:10.1021/acsnano.7b01023

144. Lam P-L, Wong W-Y, Bian Z, Chui C-H GR. Recent advances in green nanoparticulate systems for drug delivery: efficient delivery and safety concern. Nanomedicine. 2017;12(4):357-385. doi:10.2217/nnm-2016-0305

145. Teimouri M, Nia AH, Abnous K, Eshghi H, Ramezani M. Graphene oxide-cationic polymer conjugates: synthesis and application as gene delivery vectors. Plasmid. 2016;84:51-60. doi:10.1016/j.plasmid.2016.03.002

146. Zhou X, Laroche F, Lamers GE, et al. Ultra-small graphene oxide functionalized with polyethylenimine (PEI) for very efficient gene delivery in cell and zebrafish embryos. Nano Res. 2012;5 (10):703-709. doi:10.1007/s12274-012-0254-x

147. $\mathrm{Hu} \mathrm{H}$, Tang C, Yin C. Folate conjugated trimethyl chitosan/ graphene oxide nanocomplexes as potential carriers for drug and gene delivery. Mater Lett. 2014;125:82-85. doi:10.1016/j. matlet.2014.03.133

148. Zhu X, Zhang Y, Huang H, Zhang H, Hou L, Zhang Z. Functionalized graphene oxide-based thermosensitive hydrogel for near-infrared chemo-photothermal therapy on tumor. J Biomater Appl. 2016;30(8):1230-1241. doi:10.1177/0885328 215619583

149. Bao H, Pan Y, Ping Yet al., Chitosan-functionalized graphene oxide as a nanocarrier for drug and gene delivery. Small. 2011;7 (11):1569-1578. doi:10.1002/smll.201100191

150. Liu X, Ma D, Tang H, et al. Polyamidoamine dendrimer and oleic acid-functionalized graphene as biocompatible and efficient gene delivery vectors. ACS Appl Mater Interfaces. 2014;6 (11):8173-8183. doi:10.1021/am500812h

151. Kou Z, Wang X, Yuan R, et al. A promising gene delivery system developed from PEGylated MoS 2 nanosheets for gene therapy. Nanoscale Res Lett. 2014;9(1):587. doi:10.1186/1556-276X-9-587

152. Feng L, Zhang S, Liu Z. Graphene based gene transfection. Nanoscale. 2011;3(3):1252-1257. doi:10.1039/c0nr00680g

153. Cao X, Zheng S, Zhang S, et al. Functionalized graphene oxide with hepatocyte targeting as anti-tumor drug and gene intracellular transporters. J Nanosci Nanotechnol. 2015;15(3):2052-2059. doi:10.1166/jnn.2015.9145

154. Fan Z, Fu PP, Yu H, Ray PC. Theranostic nanomedicine for cancer detection and treatment. J Food Drug Anal. 2014;22 (1):3-17. doi:10.1016/j.jfda.2014.01.001

155. Fan Z, Senapati D, Singh AK, Ray PC. Theranostic magnetic core-plasmonic shell star shape nanoparticle for the isolation of targeted rare tumor cells from whole blood, fluorescence imaging, and photothermal destruction of cancer. Mol Pharm. 2013;10 (3):857-866. doi:10.1021/mp300468q

156. Li J, Lyv Z, Li Y, et al. A theranostic prodrug delivery system based on Pt (IV) conjugated nano-graphene oxide with synergistic effect to enhance the therapeutic efficacy of $\mathrm{Pt}$ drug. Biomaterials. 2015;51:12-21. doi:10.1016/j.biomaterials.2015 .01 .074 
157. Gorgizadeh M, Azarpira N, Sattarahmady N. In vitro and in vivo tumor annihilation by near-infrared photothermal effect of a NiFe2O4/C nanocomposite. Colloids Surf B Biointerfaces. 2018;170:393-400.

158. Luo Y, Li Z, Zhu C, et al. Graphene-like metal-free 2D nanosheets for cancer imaging and theranostics. Trends Biotechnol. 2018;36 (11):1145-1156. doi:10.1016/j.tibtech.2018.05.012

159. Chen H, Liu F, Lei Z, Ma L, Wang Z. Fe2O3@Au core@shell nanoparticle-graphene nanocomposites as theranostic agents for bioimaging and chemo-photothermal synergistic therapy. RSC $A d v$. 2015;5(103):84980-84987. doi:10.1039/C5RA17143A

160. Bahreyni A, Yazdian-Robati R, Hashemitabar S, et al. A new chemotherapy agent-free theranostic system composed of graphene oxide nano-complex and aptamers for treatment of cancer cells. Int $J$ Pharm. 2017;526(1-2):391-399. doi:10.1016/j. ijpharm.2017.05.014

161. Yang K, Hu L, Ma X, et al. Multimodal imaging guided photothermal therapy using functionalized graphene nanosheets anchored with magnetic nanoparticles. Adv Mater. 2012;24 (14):1868-1872. doi:10.1002/adma.201104964

162. Lamb J, Fischer E, Rosillo-Lopez M, Salzmann CG, Holland JP. Multi-functionalised graphene nanoflakes as tumour-targeting theranostic drug-delivery vehicles. Chem Sci. 2019;10 (38):8880-8888. doi:10.1039/C9SC03736E

163. Karki N, Rana A, Tiwari H, Negi P, Sahoo NG. Theranostics application of graphene-based materials in cancer imaging, targeting and treatment. In: Lasfar A, Cohen-Solal K, editors. Tumor Progression and Metastasis. IntechOpen; 2020.

164. Janib SM, Moses AS, MacKay JA. Imaging and drug delivery using theranostic nanoparticles. Adv Drug Deliv Rev. 2010;62 (11):1052-1063. doi:10.1016/j.addr.2010.08.004

165. Bao X, Yuan Y, Chen J, et al. In vivo theranostics with nearinfrared-emitting carbon dots-highly efficient photothermal therapy based on passive targeting after intravenous administration. Light. 2018;7(1):1-11. doi:10.1038/s41377-018-0090-1

166. Pramanik A, Chavva SR, Fan Z, Sinha SS, Nellore BPV, Ray PC. Extremely high two-photon absorbing graphene oxide for imaging of tumor cells in the second biological window. $J$ Phys Chem Lett. 2014;5(12):2150-2154. doi:10.1021/jz5009856

167. Yang Y, Wang S, Wang C, Tian C, Shen Y, Zhu M. Engineered targeted hyaluronic acid-glutathione-stabilized gold nanoclusters/ graphene oxide-5-fluorouracil as a smart theranostic platform for stimulus-controlled fluorescence imaging-assisted synergetic chemo/phototherapy. Chem Asian J. 2019;14(9):1418-1423. doi:10.1002/asia.201900153

168. Prasad R, Yadav AS, Gorain M, et al. Graphene oxide supported liposomes as red emissive theranostics for phototriggered tissue visualization and tumor regression. ACS Appl Bio Mater. 2019;2 (8):3312-3320. doi:10.1021/acsabm.9b00335

169. Kumawat MK, Thakur M, Bahadur R, et al. Preparation of graphene oxide-graphene quantum dots hybrid and its application in cancer theranostics. Mater Sci Eng C. 2019;103:109774. doi:10.1016/j.msec.2019.109774

170. Lui CH, Mak KF, Shan J, Heinz TF. Ultrafast photoluminescence from graphene. Phys Rev Lett. 2010;105(12):127404. doi:10.1103/PhysRevLett.105.127404

171. Xu X, Liu C, Sun Z, et al. Interfacial engineering in graphene bandgap. Chem Soc Rev. 2018;47(9):3059-3099.

172. Xiong J, Yang Q, Li J, Zhou S. Effects of MDM2 inhibitors on vascular endothelial growth factor-mediated tumor angiogenesis in human breast cancer. Angiogenesis. 2014;17(1):37-50. doi:10.1007/s10456-013-9376-3

173. Khurana R, Simons M, Martin JF, Zachary IC. Role of angiogenesis in cardiovascular disease: a critical appraisal. Circulation. 2005;112 (12):1813-1824. doi:10.1161/CIRCULATIONAHA.105.535294
174. Carmeliet P, Jain RK. Angiogenesis in cancer and other diseases. nature. 2000;407(6801):249-257. doi:10.1038/35025220

175. Mukherjee S, Sriram P, Barui AK, et al. Graphene oxides show angiogenic properties. Adv Healthcare Mater. 2015;4 (11):1722-1732. doi:10.1002/adhm.201500155

176. Qian Y, Song J, Zhao X, et al. 3D fabrication with integration molding of a graphene oxide/polycaprolactone nanoscaffold for neurite regeneration and angiogenesis. Advan Sci. 2018;5 (4):1700499. doi:10.1002/advs.201700499

177. Sun Z, Huang P, Tong G, et al. VEGF-loaded graphene oxide as theranostics for multi-modality imaging-monitored targeting therapeutic angiogenesis of ischemic muscle. Nanoscale. 2013;5 (15):6857-6866. doi:10.1039/c3nr01573d

178. Paul A, Hasan A, Kindi HA, et al. Injectable graphene oxide/ hydrogel-based angiogenic gene delivery system for vasculogenesis and cardiac repair. ACS Nano. 2014;8(8):8050-8062. doi: $10.1021 / \mathrm{nn} 5020787$

179. Xue D, Chen E, Zhong H, et al. Immunomodulatory properties of graphene oxide for osteogenesis and angiogenesis. Int J Nanomedicine. 2018;13:5799. doi:10.2147/IJN.S170305

180. Ur Rehman SR, Augustine R, Zahid AA, et al. Reduced graphene oxide incorporated GelMA hydrogel promotes angiogenesis for wound healing applications. Int $J$ Nanomedicine. 2019;14 (9603):9603-9617. doi:10.2147/IJN.S218120

181. Shim G, Kim J-Y, Han J, et al. Reduced graphene oxide nanosheets coated with an anti-angiogenic anticancer low-molecular-weight heparin derivative for delivery of anticancer drugs. $J$ Controlled Release. 2014;189:80-89. doi:10.1016/j.jconrel.2014.06.026

182. Lai P-X, Chen C-W, Wei S-C, et al. Ultrastrong trapping of VEGF by graphene oxide: anti-angiogenesis application. Biomaterials. 2016;109:12-22. doi:10.1016/j.biomaterials.2016.09.005

183. Cibecchini G, Veronesi M, Catelani T, Bandiera T, Guarnieri D, Pompa PP. Antiangiogenic effect of graphene oxide in primary human endothelial cells. ACS Appl Mater Interfaces. 2020;12 (20):22507-22518.

184. Wierzbicki M, Sawosz E, Strojny B, Jaworski S, Grodzik M, Chwalibog A. NF-kB-related decrease of glioma angiogenic potential by graphite nanoparticles and graphene oxide nanoplatelets. Sci Rep. 2018;8(1):1-9. doi:10.1038/s41598-018-33179-3

185. Li J, Ge X, Cui C, et al. Preparation and characterization of functionalized graphene oxide carrier for siRNA delivery. Int J Mol Sci. 2018;19(10):3202. doi:10.3390/ijms19103202

186. Stone V, Johnston H, Schins RPF. Development of in vitro systems for nanotoxicology: methodological considerations. Crit Rev Toxicol. 2009;39(7):613-626. doi:10.1080/10408440903120975

187. Liu K, Zhang -J-J, Cheng -F-F, Zheng -T-T, Wang C, Zhu -J-J. Green and facile synthesis of highly biocompatible graphene nanosheets and its application for cellular imaging and drug delivery. J Mater Chem. 2011;21(32):12034-12040. doi:10.1039/c1jm10749f

188. Sanchez VC, Pietruska JR, Miselis NR, Hurt RH, Kane AB. Biopersistence and potential adverse health impacts of fibrous nanomaterials: what have we learned from asbestos? Wiley Interdiscip Rev Nanomed Nanobiotechnol. 2009;1(5):511-529.

189. Dreyer DR, Park S, Bielawski CW, Ruoff RS. The chemistry of graphene oxide. Chem Soc Rev. 2010;39(1):228-240. doi:10.1039/ B917103G

190. Muthoosamy K, Abubakar IB, Bai RG, Loh H-S, Manickam S. Exceedingly higher co-loading of curcumin and paclitaxel onto polymer-functionalized reduced graphene oxide for highly potent synergistic anticancer treatment. Sci Rep. 2016;6:32808. doi: $10.1038 /$ srep32808

191. Cho YC, Pak PJ, Joo YH, Lee H-S CN. In vitro and in vivo comparison of the immunotoxicity of single- and multi-layered graphene oxides with or without pluronic F-127. Sci Rep. 2016;6:38884. doi:10.1038/srep38884 
192. Zhao M-L, Liu X-Q, Cao Y, et al. Enhancement of interaction of L-929 cells with functionalized graphene via $\mathrm{COOH}+$ ion implantation vs. chemical method. Sci Rep. 2016;6:37112. doi:10.1038/ srep37112

193. Vuppaladadium SSR, Agarwal T, Kulanthaivel S, et al. Silanization improves biocompatibility of graphene oxide. Mater Sci Eng C. 2020;110:110647. doi:10.1016/j.msec.2020.110647

194. Sydlik SA, Jhunjhunwala S, Webber MJ, Anderson DG, Langer R. In vivo compatibility of graphene oxide with differing oxidation states. ACS Nano. 2015;9(4):3866-3874. doi:10.1021/ acsnano. 5 b01290

195. Zhang X, Yin J, Peng C, et al. Distribution and biocompatibility studies of graphene oxide in mice after intravenous administration. carbon. 2011;49(3):986-995. doi:10.1016/j. carbon.2010.11.005

196. Nurunnabi M, Khatun Z, Huh KM, et al. In vivo biodistribution and toxicology of carboxylated graphene quantum dots. ACS Nano. 2013;7(8):6858-6867. doi:10.1021/nn402043c
197. Yang K, Zhang S, Zhang G, Sun X, Lee S-T LZ. Graphene in mice: ultrahigh in vivo tumor uptake and efficient photothermal therapy. Nano Lett. 2010;10(9):3318-3323. doi:10.1021/ nl100996u

198. Reina G, González-Domínguez JM, Criado A, Vázquez E, Bianco A, Prato M. Promises, facts and challenges for graphene in biomedical applications. Chem Soc Rev. 2017;46 (15):4400-4416. doi:10.1039/C7CS00363C

199. Bianco A. Graphene: safe or toxic? The two faces of the medal. Angewandte Chemie Int Ed. 2013;52(19):4986-4997. doi:10.1002/anie.201209099

200. Zhang Y, Ali SF, Dervishi E, et al. Cytotoxicity effects of graphene and single-wall carbon nanotubes in neural phaeochromocytoma-derived PC12 cells. ACS Nano. 2010;4 (6):3181-3186. doi:10.1021/nn1007176

\section{Publish your work in this journal}

The International Journal of Nanomedicine is an international, peerreviewed journal focusing on the application of nanotechnology in diagnostics, therapeutics, and drug delivery systems throughout the biomedical field. This journal is indexed on PubMed Central, MedLine, CAS, SciSearch ${ }^{\mathbb{}}$, Current Contents ${ }^{\mathbb{R}} /$ Clinical Medicine,
Journal Citation Reports/Science Edition, EMBase, Scopus and the Elsevier Bibliographic databases. The manuscript management system is completely online and includes a very quick and fair peer-review system, which is all easy to use. Visit http://www.dovepress.com/ testimonials.php to read real quotes from published authors. 